University of Wollongong

Research Online

Faculty of Engineering and Information

Faculty of Engineering and Information

Sciences - Papers: Part A

Sciences

2017

Stacking fault tetrahedron induced plasticity in copper single crystal

Liang Zhang

University of Wollongong, liangz@uow.edu.au

Cheng Lu

University of Wollongong, chenglu@uow.edu.au

Anh Kiet Tieu

University of Wollongong, ktieu@uow.edu.au

Lihong Su

University of Wollongong, lihongsu@uow.edu.au

Xing Zhao

University of Wollongong, xz920@uowmail.edu.au

See next page for additional authors

Follow this and additional works at: https://ro.uow.edu.au/eispapers

Part of the Engineering Commons, and the Science and Technology Studies Commons

Research Online is the open access institutional repository for the University of Wollongong. For further information contact the UOW Library: research-pubs@uow.edu.au 


\title{
Stacking fault tetrahedron induced plasticity in copper single crystal
}

\begin{abstract}
Stacking fault tetrahedron (SFT) is the most common type of vacancy clustered defects in fcc metals and alloys, and can play an important role in the mechanical properties of metallic materials. In this study, molecular dynamics (MD) simulations were carried out to investigate the incipience of plasticity and the underlying atomic mechanisms in copper single crystals with SFT. Different deformation mechanisms of SFT were reported due to the crystal orientations and loading directions (compression and tension). The results showed that the incipient plasticity in crystals with SFT resulted from the heterogeneous dislocation nucleation from SFT, so the stress required for plastic deformation was less than that needed

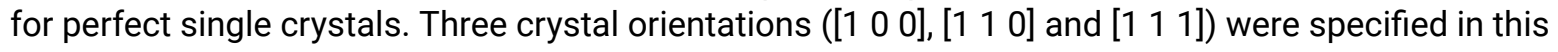
study because they can represent most of the typical deformation mechanisms of SFT. MD simulations revealed that the structural transformation of SFT was frequent under the applied loading; a metastable SFT structure and the collapse of SFT were usually observed. The structural transformation resulted in a different reduction of yield stress in compression and tension, and also caused a decreased or reversed compression/tension asymmetry. Compressive stress can result in the unfaulting of Frank loop in some crystal orientations. According to the elastic theory of dislocation, the process of unfaulting was closely related to the size of the dislocation loop and the stacking fault energy.
\end{abstract}

\section{Disciplines}

Engineering | Science and Technology Studies

\section{Publication Details}

Zhang, L., Lu, C., Tieu, K., Su, L., Zhao, X. \& Pei, L. (2017). Stacking fault tetrahedron induced plasticity in copper single crystal. Materials Science and Engineering A: Structural Materials: Properties, Microstructure and Processing, 680 27-38.

\section{Authors}

Liang Zhang, Cheng Lu, Anh Kiet Tieu, Lihong Su, Xing Zhao, and Linqing Pei 


\title{
Stacking fault tetrahedron induced plasticity in copper single crystal
}

\author{
Liang Zhang ${ }^{1}$, Cheng $\mathrm{Lu}^{1}{ }^{*}$, Kiet Tieu ${ }^{1}$, Lihong $\mathrm{Su}^{1}$, Xing Zhao ${ }^{1}$, Linqing Pei ${ }^{2}$ \\ ${ }^{1}$ School of Mechanical, Materials and Mechatronic Engineering, University of Wollongong, \\ Wollongong, NSW 2522, Australia. \\ ${ }^{2}$ Department of Mechanical Engineering, Chongqing University, Chongqing, 400044, China \\ *Corresponding author. Tel.:+6142214639; fax:+61242213101; \\ E-mail address: chenglu@uow.edu.au (C. Lu) or lz592@uowmail.edu.au ((L. Zhang)
}

\begin{abstract}
Stacking fault tetrahedron (SFT) is the most common type of vacancy clustered defects in fcc metals and alloys, and can play an important role in the mechanical properties of metallic materials. In this study, molecular dynamics (MD) simulations were carried out to investigate the incipience of plasticity and the underlying atomic mechanisms in copper single crystals with SFT. Different deformation mechanisms of SFT were reported due to the crystal orientations and loading directions (compression and tension). The results showed that the incipient plasticity in crystals with SFT resulted from the heterogeneous dislocation nucleation from SFT, so the stress required for plastic deformation was less than that needed for

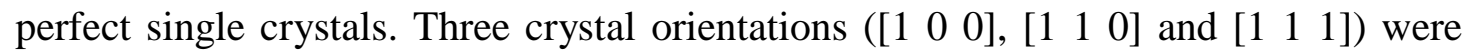
specified in this study because they can represent most of the typical deformation mechanisms of SFT. MD simulations revealed that the structural transformation of SFT was frequent under the applied loading; a metastable SFT structure and the collapse of SFT were usually observed. The structural transformation resulted in a different reduction of yield stress in compression and tension, and also caused a decreased or reversed compression/tension asymmetry. Compressive stress can result in the unfaulting of Frank loop in some crystal orientations. According to the elastic theory of dislocation, the process of unfaulting was closely related to the size of the dislocation loop and the stacking fault energy.
\end{abstract}

Keywords: Molecular dynamics; Stacking fault tetrahedron; Dislocation; Plasticity; Structural transformation 


\section{Introduction}

The propagation and interaction of lattice dislocations within the grains is fundamentally important for the plastic deformation of conventional coarsened metallic materials, but dislocations in nanocrystalline metallic materials are less likely to be present because at very small grain sizes, it is less likely that the dislocation loops will be stable[1, 2]. In a small enough volume (at nanometer-scale) of a material with limited pre-existing dislocations, the nucleation of new dislocations may dominate the deformation mechanism of the incipient plasticity, rather than the propagation, multiplication or interaction of the initial dislocations[3-6]. Generally, a dislocation can nucleate in small volumes from perfect lattice homogeneously or from structural defects heterogeneously. For a sample free from defects, the nucleation of dislocation occurs randomly throughout the volume[7, 8]; but this deformation mechanism needs very large stresses, whereas heterogeneous nucleation of dislocation usually occurs with crystal defects, such as point defects[9, 10], voids[11, 12], free surfaces[13, 14] and grain boundaries[15-19]. These defects act as stress concentrators that emit dislocations at stresses less than that required for homogeneous dislocation nucleation.

Among different point defects (e.g. single or di-vacancies, interstitial or impurity atoms), stacking fault tetrahedron (SFT) is the common one that formed in fcc crystals under various circumstances. This special shape of vacancy cluster was first observed in quenched gold [20]. Silcox and Hirsch reported that the SFT was generated from a faulted loop that clustered by a number of quenched-in vacancies. The faulted loop then evolved to a tetrahedron-shaped defect in which the edges were consisted of stair rod dislocations while the closed planes were organized by stacking faults. Later, SFTs were observed in some deformed metals and alloys at room and higher temperatures[21]. SFTs were subsequently reported in electron-irradiated metals[22] using high voltage electron microscopy, while recently, SFTs have been observed in the Ni-based superalloy that experienced low cycle fatigue by controlling the strain rate at high temperatures[23]. In addition to experimental observation, this type of 
vacancy defect has also been modeled by using molecular dynamics (MD) simulations[24-28]. Based on these experimental and simulation results, Loretto et al.[29] concluded there were three fundamental mechanisms for producing SFT; (i) the vacancies accumulation based Silcox-Hirsch mechanism; (ii) various mechanisms based on the glide and cross slip of dislocations, and (iii) the merging of glide elements followed by growth. In their experiment, Matsukawa and Zinkle[30] observed the one-dimensional fast motion of nanometer-sized vacancy clusters and proposed that this motion may be a key physical mechanism for the self-organization of nanometer-sized SFT arrays.

Besides those studies on the formation mechanisms of SFT, many recent research interests now focus on the interaction of SFT with other structural defects, mainly dislocations[31-38] and twin boundary[39-41]. For example, previous MD studies revealed that SFT can provide a strong obstacle for glide dislocations that ultimately increase the yield strength and decrease ductility[42, 43]. Robach et al.[33] investigated the interaction of an SFT with an edge dislocation in copper and gold by using in situ transmission electron microscope (TEM) and MD simulations. They found that this interaction can result in the SFT being converted to another type of defect, be annihilated, or be sheared into two defects. Lee and Wirth[35] used MD simulations to study the interaction between SFT and a mixed dislocation in copper and noted that depending on the interaction geometry, it can result in destabilization, partial absorption, shearing, or a simple bypass of the SFT. By using MD simulations, Niewczas and Hoagland[39] studied the interaction of moving twin boundaries with SFT and found that this interaction generally damaged the parent SFT and the formation of new defects in the twin lattice. Yu et al.[40] observed the radiation induced migration of twin boundaries and found that the migrating twin boundaries played a prominent role in removing the radiation-induced SFTs.

While previous literatures are mostly limited to the formation of SFT or their interaction with other structural defects, very few studies focused on the effect of SFT on the mechanical response of materials with small grain size where dislocations are 
unlikely to be present, even though SFT can significantly affect the mechanical property of materials with incipient plasticity. For example, the atomistic simulations of nanoindentation tests showed that SFT reduced the pressure needed to nucleate a dislocation by almost half that of a perfect crystal[44]; this means the governing deformation mechanisms must be explored in the presence of this defect. Salehinia and Bahr[45] performed MD simulations in a nanoindentation test of copper single crystal to investigate how the orientation of SFT affects the load needed to initiate plastic deformation; their results indicated that a downward SFT affected the mechanical behavior of a copper single crystal much more than an upward orientated SFT. In addition, their recent MD study indicated that the presence of SFT can decrease or even reverse the compression/tension $(\mathrm{C} / \mathrm{T})$ asymmetry of the copper single crystal[46]. Although in the above studies some atomic configurations were present at the onset of plasticity, there was no concise atomic picture of the dislocation mechanisms that actually induced the incipience of plasticity, which means that some remaining issues need further discussion. For example, what is the deformation mechanism that result in the different reduction in yield stress under tension and compression? Why can the $\mathrm{C} / \mathrm{T}$ asymmetry decrease or reverse in the presence of SFT? How does the structural transformation of SFT occur during the applied loading? To the best of the authors' knowledge, the underlying mechanisms related to these behaviors have not been reported, so our objective was to obtain an atomic insight into the underlying dislocation mechanisms of SFT, which is needed to understand the incipience of plasticity in small volume crystal induced by SFT.

In this work, molecular dynamics simulations were performed on copper single crystal with SFT under either uniaxial tension or compression, and with a variety of crystal orientations. Copper single crystals with a perfect structural model were also investigated in the same simulation conditions for comparison. The simulation methods were introduced in Section 2. Mechanical response and the deformation mechanisms of the simulation samples were presented in Section 3. The structural transformation of SFTs and the collapse of SFTs were observed during the applied 
loading, these mechanisms were found closely related to the higher reduction in the yield stress in compression and result in a decreased or reversed C/T asymmetry. In addition, the structural conversion from a sessile Frank partial loop to a glissile perfect loop was also observed in this study; the simulation results were compared with the previous experimental observations and discussed in Section 4.

\section{Simulation method}

Molecular dynamics simulations were carried out by using the parallel molecular dynamics code LAMMPS[47]. The interatomic potential used for simulations plays a significant role in accurately predicting the structural defects. In this study, copper was modeled using the embedded-atom method (EAM) potential developed by Mishin et al.[48]. The well defined potential can fit a large set of first-principles and experimental data. For example, the intrinsic stacking fault energy and unstable stacking fault energy of copper from previous simulation result are $44.6 \mathrm{~mJ} / \mathrm{m}^{2}$ and $173.4 \mathrm{~mJ} / \mathrm{m}^{2}$ respectively[49], which are comparable with the experimental measurement $45 \mathrm{~mJ} / \mathrm{m}^{2}$ and $162 \mathrm{~mJ} / \mathrm{m}^{2}[48,50]$. The visualization tools and sophisticated automated dislocation detection techniques have improved immensely in recent years, so we took full advantage of these techniques to obtain information at an atomic scale. Atomeye[51] and OVITO[52] were used as visualization tools to illustrate the simulation models. The common neighbor analysis (CNA) method[53] was used to highlight atoms that without perfect fcc structures. This local atomic classification scheme allows defect structures and their evolution to be easily identified during the simulation. Dislocation extraction algorithm (DXA)[54, 55] was used to give quantitative analysis and a detailed investigation of dislocations. The robust code can identify a wide range of dislocation types and extract dislocation lines in a fully automated way. Also, the Burgers vectors of different dislocations can be calculated.

In order to investigate the influence of SFT on the incipience of plasticity of a single crystal, perfect single crystals with various crystal orientations were constructed and then an SFT was placed at the centre of each crystal. Periodic 
boundary conditions were applied to the simulation model in all directions. The simulation box was approximately $20 \times 20 \times 20 \mathrm{~nm}^{3}$, and contained approximately $6.7 \times 10^{5}$ atoms. It was thought that a $16^{3} \mathrm{~nm}^{3}$ cell would be large enough to avoid any significant effects on the dislocation nucleation dynamics that caused by periodic boundaries[7]. This meant that any further increase of the model size does not essentially affect the stress that required for the incipience of crystal plasticity. In this study, the SFT defect was generated based on the Silcox-Hirsch mechanism[20], which indicated that a platelet of vacancies can collapse to form a Frank partial dislocation loop and then evolve into an SFT. So we referenced the previous method[45] and removed a triangular Frank loop platelet of vacancies in the (1 111 ) plane. The triangular platelet contained 55 vacancies, 10 on each edge, corresponding to an SFT with $2.3 \mathrm{~nm}$ long edge that was similar to the size of SFTs in copper observed in the experiments[56]. The density of SFT in this modeling was approximately $10^{24} \mathrm{~m}^{-3}$, which was close to the $10^{23} \mathrm{~m}^{-3}$ reported for copper single crystal[57].
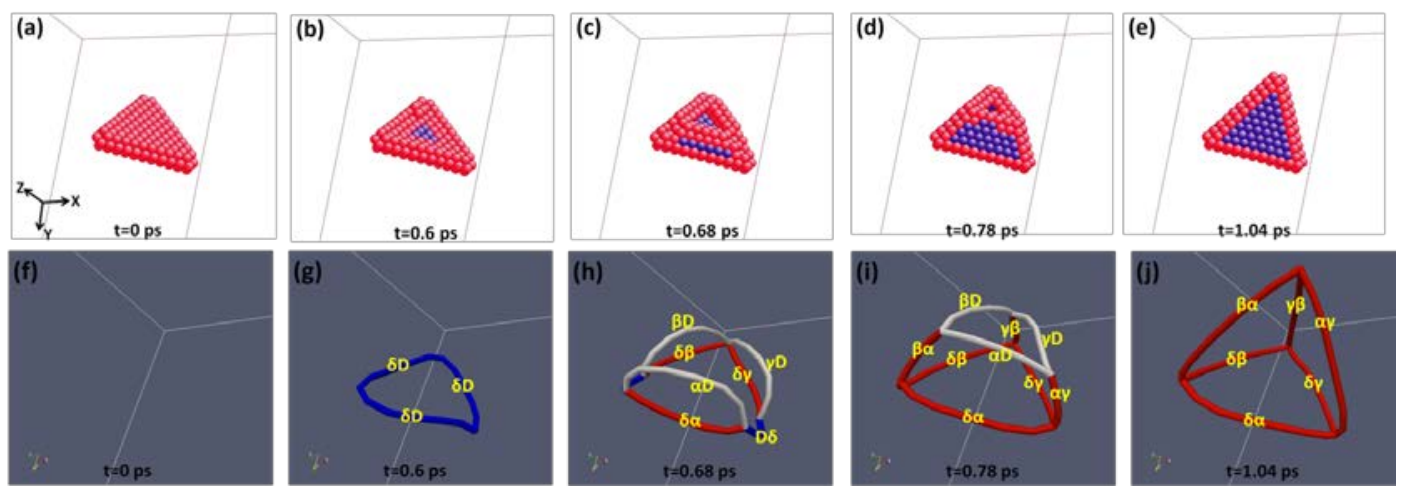

Fig.1 Snapshots to illustrate the formation process of an SFT from a vacancy triangular platelet based on the Silcox-Hirsch mechanism. (a)-(e) show the results from MD simulations; (f)-(j) show the extracted dislocation segment by using the dislocation extraction algorithm. (Colour online)

The simulation system was initially relaxed for $10 \mathrm{ps}$ using the isobaric-isothermal (NPT) ensemble at a temperature of $10 \mathrm{~K}$ and a pressure of 0 bar. Fig. 1 shows five snapshots from the MD simulation (a-e) and the results of the dislocation extraction (f-j) to illustrate how an SFT was generated from a vacancy triangular platelet. Here, the atoms with a perfect fcc structure were removed to make the defects easier to see. 
The times denote the time that elapsed from when the vacancies were first introduced. In the first $0.6 \mathrm{ps}$, a closed Frank partial dislocation loop was produced after a platelet of vacancies collapsed, as shown in Fig.1(g). From an atomistic perspective, this process can be regarded as the collective displacement of atoms within the tetrahedra as they gradually dropped into the hole left by the original vacancy platelet[24] (see Fig.1(b)). Generally, this is called a negative Frank dislocation while for a positive one, the Frank dislocation loop was regarded to generate by the precipitation of interstitial atoms on a closely packed platelet[58]. Both of the positive and negative Frank loops contain stacking faults, while a stacking fault will be stable if the fault energy is low enough; but this was not the case for copper in this study (about 44.4 $\mathrm{mJ} / \mathrm{m}^{2}$ [48]). Simulation results show that the Frank partial soon dissociated into a stair-rod dislocation with lower energy and a Shockley partial dislocation on an intersecting slip plane (see Fig.1(c) and (h)). This process is according to a reaction of the type:

$$
\begin{aligned}
& \delta \mathrm{D} \rightarrow \delta \alpha+\alpha \mathrm{D} \quad(1 / 3)\left[\begin{array}{lll}
1 & 1 & 1
\end{array}\right] \rightarrow(1 / 6)\left[\begin{array}{lll}
1 & 0 & 1
\end{array}\right]+(1 / 6)\left[\begin{array}{lll}
1 & 2 & 1
\end{array}\right] \\
& \delta \mathrm{D} \rightarrow \delta \gamma+\gamma \mathrm{D} \quad(1 / 3)\left[\begin{array}{lll}
1 & 1 & 1
\end{array}\right] \rightarrow(1 / 6)\left[\begin{array}{lll}
0 & 1 & 1
\end{array}\right]+(1 / 6)\left[\begin{array}{lll}
2 & 1 & 1
\end{array}\right] \\
& \delta \mathrm{D} \rightarrow \delta \beta+\beta \mathrm{D} \quad(1 / 3)\left[\begin{array}{lll}
1 & 1 & 1
\end{array}\right] \rightarrow(1 / 6)\left[\begin{array}{lll}
1 & 1 & 0
\end{array}\right]+(1 / 6)\left[\begin{array}{lll}
1 & 1 & 2
\end{array}\right]
\end{aligned}
$$

The dislocations and slip planes are described using Thompson tetrahedra[20] notation and the definition given by Hirth and Lothe[59]. Fig.1(d) and (i) show that when the propagated Shockley partial dislocations met each other, they soon reacted to form another set of stair-rod dislocations. This process can be described by the reaction where:

$$
\begin{aligned}
& \alpha \mathrm{D}+\mathrm{D} \gamma \rightarrow \alpha \gamma \quad(1 / 6)\left[\begin{array}{lll}
1 & 2 & 1
\end{array}\right]+(1 / 6)\left[\begin{array}{lll}
\overline{2} & \overline{1} & \overline{1}
\end{array}\right] \rightarrow(1 / 6)\left[\begin{array}{lll}
\overline{1} & 1 & 0
\end{array}\right]
\end{aligned}
$$

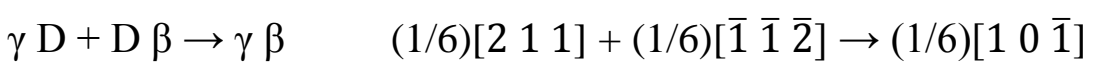

$$
\begin{aligned}
& \beta \mathrm{D}+\mathrm{D} \alpha \rightarrow \beta \alpha \quad(1 / 6)\left[\begin{array}{lll}
1 & 1 & 2
\end{array}\right]+(1 / 6)\left[\begin{array}{lll}
\overline{1} & \overline{2} & \overline{1}
\end{array}\right] \rightarrow(1 / 6)\left[\begin{array}{lll}
0 & \overline{1} & 1
\end{array}\right]
\end{aligned}
$$

The Shockley partials glided towards the apex of the tetrahedra and finally constructed a tetrahedron with intrinsic stacking faults on $\{111\}$ planes and $1 / 6<110>$ type stair-rod dislocations along the edges, as shown in Fig.1(e) and (j). The entire 
process of SFT formation takes about 1 ps.

After obtaining an equilibrium structure of SFT and the initial relaxation of the simulation system, either uniaxial tensile or compressive loading with a constant strain rate of $1 \times 10^{8} \mathrm{~s}^{-1}$ was applied along $\mathrm{Y}$ axis (which is defined as the crystal orientation in this study) on the simulated sample. Previous simulations by Spearot et al.[60] shows that the strain rate $\left(10^{7} \sim 10^{9} / \mathrm{s}\right)$ does not affect the elastic modulus of the single crystal sample and plays a minor role in the maximum tensile stress. Therefore, the specified strain rate of $10^{8} / \mathrm{s}$ used in this study is capable of capturing the essential properties of the simulation samples and avoid any significant rate influenced behavior. During the deformation, the stresses along $\mathrm{X}$ and $\mathrm{Z}$ axis were kept at zero by using the NPT ensemble. The simulations were conducted at a temperature of $10 \mathrm{~K}$ to avoid thermal disturbance of atoms at higher temperatures and provide a clearer view on the evolution of SFT structures and the corresponding dislocation activities. An integration time step was set as 1 fs throughout the MD simulations. For the mechanical response, the system stress was attained by calculating the pressure of the entire system of atoms, while system strain was derived from the positions of the periodic boundaries along loading direction.

\section{Results}

\subsection{Compressive and tensile stress response}

The yield stress of perfect crystals and crystals with SFT for different orientations are listed in Table.1. The yield stress in this study is defined as the maximum tensile or compressive stress, and it corresponds to the incipience of plasticity. A detailed analysis of three crystal orientations ([l $\left.\begin{array}{lll}1 & 0 & 0\end{array}\right],\left[\begin{array}{lll}1 & 1 & 0\end{array}\right]$ and $\left.\left[\begin{array}{lll}1 & 1 & 1\end{array}\right]\right)$ are present, because it is found that the three selected cases can represent most of the typical stress response and deformation mechanisms of different oriented SFT. The mechanical

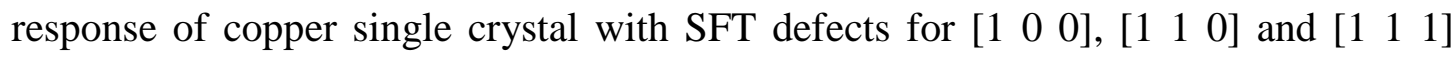
orientations under uniaxial loading at $10 \mathrm{~K}$ are shown in Fig.2. The stress-strain curves of perfect crystals are also plotted for comparison.

In the elastic stage, either elastic hardening or softening occurred in different 
loading conditions. The elastic hardening and elastic softening defined here are used to describe the non-linear stress-strain curves at higher stress and strain, where the elastic modulus either exceeded or was lower than the expected value in a condition of pure linear elastic[46]. The non-linear elastic behavior is essentially due to the interatomic potential. The lowest cohesive energy of an atom occurs at its equilibrium position, and the equilibrium interatomic spacing is associated with the spacing of the first nearest atoms in $<110>$ direction $\left(\mathrm{d}_{1}=\sqrt{2} / 2 \mathrm{a}_{0} \approx 2.556 \AA\right.$, where $\mathrm{a}_{0}$ is the lattice constant). Any increasing or decreasing of the equilibrium interatomic spacing will inevitably increase the cohesive energy and result in the attraction or repulsion force between the two atoms, and this is the origin of system stress. However, the second derivative of the cohesive energy to the interatomic spacing reveals that interatomic force gradient increases under compression and decreases under tension. Therefore, by considering the dominant role of the first nearest atoms on the system stress, it is reasonable to observe the elastic hardening under compression and elastic softening under tension in most of the studied cases. It should be emphasized that this non-linear elastic behavior shows a high anisotropic. For example, a significant non-linear elastic behavior was observed in $\left[\begin{array}{lll}1 & 1 & 0\end{array}\right]$ orientation, as shown in Fig.2(c) and (d); this is also the case for [ [ $\left.8 \begin{array}{lll}8 & 7 & 1\end{array}\right]$ and [ $\left[\begin{array}{lll}4 & 3 & 1\end{array}\right]$ etc. orientations although the stress response curves are not shown here. For [1 $\left[\begin{array}{ll}1 & 0\end{array}\right]$ and [ $\left[\begin{array}{lll}1 & 1 & 1\end{array}\right]$ orientations, and for [2 2 1], [4 3 3], and [8 7 6] etc. orientations, elastic hardening and softening also occurred but is less significant. In particular, of all the studied cases, the [ $\left.\begin{array}{lll}1 & 0 & 0\end{array}\right]$ orientation shows an opposite performance, where elastic hardening occurred in tension and elastic softening occurred in compression. This is mainly due to the applied loading completely effect on the second nearest atoms in their $<1 \quad 0 \quad 0>$ direction $\left(\mathrm{d}_{2}=\mathrm{a}_{0} \approx\right.$ $3.615 \AA$ ). Therefore, in the cases that close to [ $\left.\begin{array}{lll}1 & 0 & 0\end{array}\right]$ orientation, the interatomic force between both of the first and the second nearest atoms plays a dominant role in determining the system stress and the elastic response. The unusual stress response in $\left[\begin{array}{lll}1 & 0 & 0\end{array}\right]$ orientation compared with other crystal orientations has also been reported in the previous work by Tschopp and McDowell [61]. 
(a)

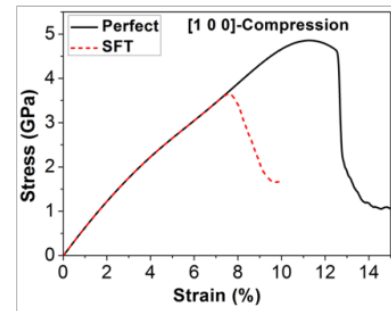

(c)

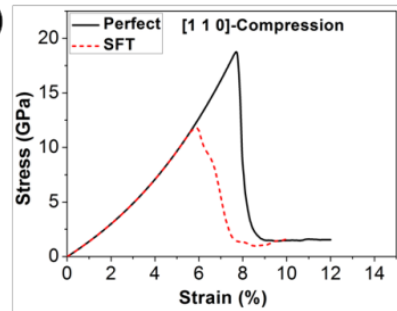

(e)

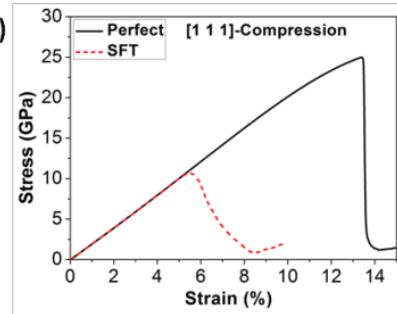

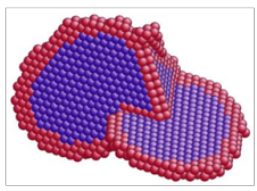

$\varepsilon=7.4 \%$
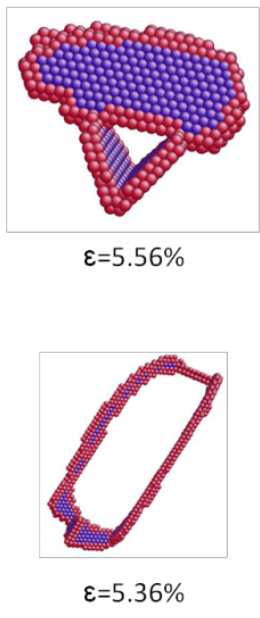

(b)

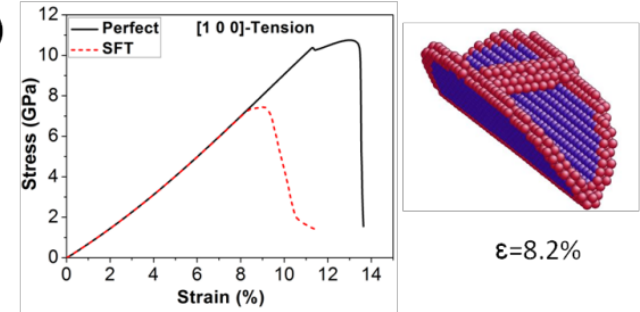

(d)
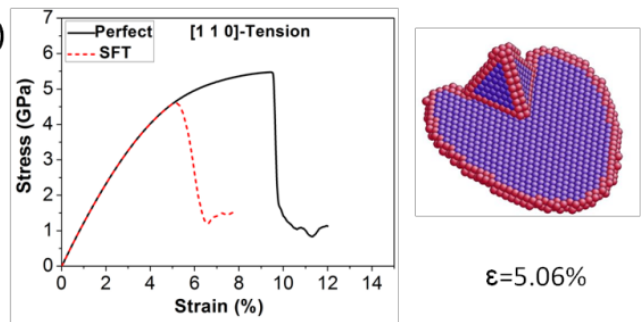

(f)

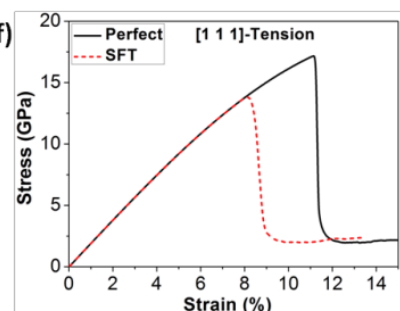

$\varepsilon=8.04 \%$

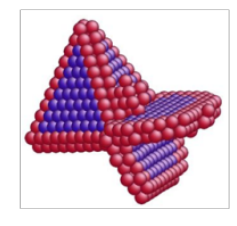

Fig.2 Stress-strain curves of perfect crystals and crystals with SFT under uniaxial compression

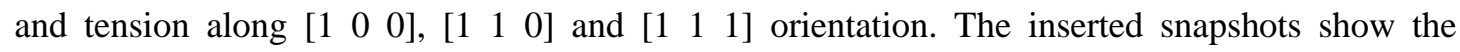
atomistic configurations of SFT at the incipient of plasticity. The red atoms represent the dislocation core and the blue atoms represents the stacking fault. (Colour online). 
Table.1 Simulation results of the perfect crystals and the crystals with an SFT under uniaxial compression (C) and tension (T) at $10 \mathrm{~K}$.

\begin{tabular}{|c|c|c|c|c|c|c|c|c|c|}
\hline Orientation & $\begin{array}{c}\text { Elastic } \\
\text { modulus (GPa) }\end{array}$ & $\begin{array}{l}\text { Yield stress } \\
\text { C-perfect } \\
\text { (GPa) }\end{array}$ & $\begin{array}{l}\text { Yield stress } \\
\text { T-perfect } \\
\text { (GPa) }\end{array}$ & $\begin{array}{l}\text { Yield stress } \\
\text { C-SFT } \\
\text { (GPa) }\end{array}$ & $\begin{array}{l}\text { Yield stress } \\
\text { T-SFT } \\
\text { (GPa) }\end{array}$ & $\begin{array}{l}\text { Reduction of } \\
\text { yield stress-C }\end{array}$ & $\begin{array}{l}\text { Reduction of } \\
\text { yield stress-T }\end{array}$ & $\mathrm{C} / \mathrm{T}$ ratio-perfect & $\mathrm{C} / \mathrm{T}$ ratio-SFT \\
\hline [100] & 65 & 4.86 & 10.75 & 3.56 & 7.44 & $24.9 \%$ & $30.8 \%$ & 0.45 & 0.48 \\
\hline [410] & 80 & 11.15 & 8.38 & 6.97 & 5.49 & $37.5 \%$ & $34.5 \%$ & 1.33 & 1.27 \\
\hline [210] & 106 & 18.42 & 6.3 & 9.76 & 4.6 & $47 \%$ & $26.9 \%$ & 2.92 & 2.12 \\
\hline [430] & 121 & 18.54 & 5.67 & 11.27 & 4.5 & $39.2 \%$ & $20.6 \%$ & 3.27 & 2.5 \\
\hline [110] & 125 & 18.76 & 5.47 & 11.79 & 4.61 & $37.1 \%$ & $15.8 \%$ & 3.43 & 2.56 \\
\hline [441] & 138 & 18.9 & 7.6 & 10.54 & 6.14 & $44.2 \%$ & $19.2 \%$ & 2.49 & 1.72 \\
\hline [221] & 163 & 18.95 & 12.62 & 8.84 & 10.08 & $53.4 \%$ & $20.1 \%$ & 1.5 & 0.88 \\
\hline [443] & 176 & 21.08 & 14.43 & 9.63 & 12.58 & $54.3 \%$ & $12.8 \%$ & 1.46 & 0.76 \\
\hline [111] & 189 & 24.97 & 17.17 & 10.67 & 13.85 & $57.3 \%$ & $19.3 \%$ & 1.45 & 0.77 \\
\hline [411] & 103 & 10.96 & 9.41 & 5.3 & 6.17 & $51.6 \%$ & $34.4 \%$ & 1.16 & 0.86 \\
\hline [211] & 153 & 15.9 & 12.47 & 7.26 & 9.76 & $54.3 \%$ & $21.7 \%$ & 1.27 & 0.74 \\
\hline [433] & 176 & 20.96 & 15.9 & 8.57 & 13.17 & $59.1 \%$ & $17.2 \%$ & 1.32 & 0.65 \\
\hline [821] & 86 & 11.58 & 8.86 & 4.85 & 5.44 & $58.1 \%$ & $38.6 \%$ & 1.31 & 0.89 \\
\hline [871] & 152 & 24.84 & 9.36 & 13.97 & 7.59 & $43.8 \%$ & $18.9 \%$ & 2.65 & 1.84 \\
\hline [876] & 180 & 22.59 & 15.66 & 8.84 & 12.81 & $60.9 \%$ & $18.2 \%$ & 1.44 & 0.69 \\
\hline [421] & 127 & 19.06 & 8.83 & 7.09 & 6.82 & $62.8 \%$ & $22.8 \%$ & 2.16 & 1.04 \\
\hline [431] & 154 & 23.26 & 10.39 & 8.85 & 8.32 & $61.9 \%$ & $19.9 \%$ & 2.24 & 1.06 \\
\hline [432] & 164 & 17.72 & 12.98 & 7.82 & 10.53 & $55.9 \%$ & $18.9 \%$ & 1.36 & 0.74 \\
\hline [321] & 150 & 18.43 & 11.32 & 7.44 & 8.54 & $59.6 \%$ & $24.5 \%$ & 1.63 & 0.87 \\
\hline
\end{tabular}


In the initial stage of plastic deformation, there is a remarkable difference in yield stress for different crystal orientations in perfect crystals and crystals with an SFT. A previous study revealed that the compressive strength in perfect copper single crystals is higher than the tensile strength for almost all crystal orientations[61]. This is also

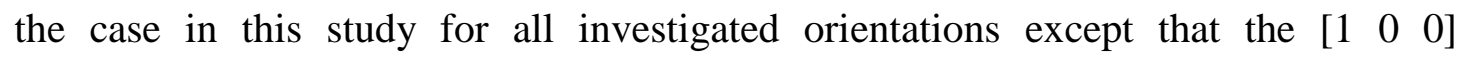
orientation has a higher yield stress in tension than in compression (see Table.1). The maximum compressive and tensile stresses are both evidenced in the [1 11 1] orientation at 24.97 GPa and 17.17 GPa respectively. As could be expected, crystals with SFT has a similar stress response as the perfect crystals, i.e. the higher loading strength in a perfect crystal should result in a higher loading strength in a crystal with SFT. Because the resolved shear stress on the closely packed plane is the same, although the yield stress is lower due to the presence of crystal defects. Indeed, the maximum tensile stress of crystal with SFT has also evidenced in [ $\left.\begin{array}{lll}1 & 1 & 1\end{array}\right]$ orientation (13.85 GPa). However, the maximum compressive stress of crystal with SFT occurs in the [8 7 1] orientation that is calculated as $13.97 \mathrm{GPa}$. Also, there is a sharp

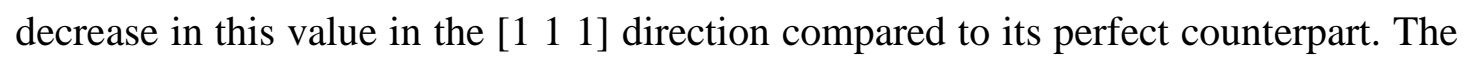
results indicate that in the presence of SFT, both of the crystal orientation and the deformation mechanisms of SFT are needed to explain the anisotropy of stress response and the incipience of plasticity for the simulated samples. The simulations revealed that once the maximum compressive or tensile stress had been reached, plasticity commenced due to the activated dislocation movement from the SFT (see atomic images inserted in Fig.2). The detailed deformation mechanisms of SFT for different crystal orientations and loading directions are specified in the next few subsections. Another remarkable feature of the stress-strain curves is the compression/tension asymmetry (different yield stress between compression and tension); this part will be discussed in Section 4 based on the deformation mechanisms of SFT.

\subsection{Deformation mechanism of SFT for [ $\left[\begin{array}{lll}1 & 0 & 0\end{array}\right]$ orientation}

The results of simulating copper single crystal with SFT under uniaxial 
compression is presented in Fig.3. Fig.3(a)-(d) shows the result obtained from the MD computer simulation, while the four selected snapshots show the SFT configurations near the yield point. The atoms were colored by the CNA parameter and then removed with the fcc structures to facilitate any defective structures; the red atoms represent the dislocation core and the blue atoms represent the stacking fault. Fig.3(e)-(h) shows the dislocations extracted from the MD results, while their Burgers vectors were computed using the dislocation extraction algorithm (DXA). A visual inspection of the MD simulation results indicated that the onset of yielding corresponded to the nucleation of partial dislocations from the SFT. The tetrahedra retained its original structure until a compressive strain equal to $\varepsilon=6.8 \%$ was applied, and then a Shockley

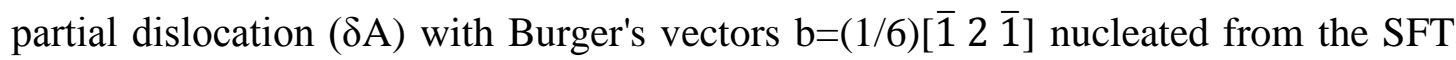
on (1 11 1) slip plane, as shown in Fig.3(b) and (f). There were three other potentially active slip planes, i.e. $\left(\begin{array}{lll}1 & 1 & \overline{1}\end{array}\right),\left(\begin{array}{lll}1 & \overline{1} & 1\end{array}\right)$, and ( $\left(\begin{array}{lll}1 & 1 & 1\end{array}\right)$. This is shown in Fig.3(c) and (g) at $\varepsilon=7.2 \%$, where the embryo dislocation loops can be seen on the four $\left\{\begin{array}{lll}1 & 1 & 1\end{array}\right\}$ planes. According to the Schmid factor analysis, they are the favored slip systems with the maximum Schmid factor $\mathrm{SF}_{(111)}^{\max }=\mathrm{SF}_{(11 \overline{1})}^{\max }=\mathrm{SF}_{(1 \overline{1} 1)}^{\max }=\mathrm{SF}_{(1 \overline{1} \overline{1})}^{\max }=0.471$. In Fig.3(d) and (h), the nucleated Shockley partial dislocation $\delta \mathrm{A}$ and $\mathrm{B} \gamma$ propagated quickly when the compressive strain reached $\varepsilon=7.4 \%$, and this finally resulted in the incipience of crystal plasticity. The nucleation process can be described by the dislocation reaction:

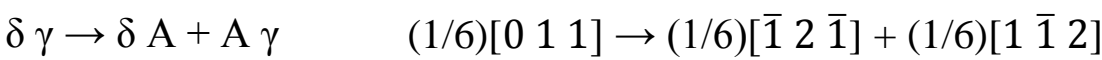

$$
\begin{aligned}
& \delta \beta \rightarrow \delta \mathrm{A}+\mathrm{A} \beta \quad(1 / 6)\left[\begin{array}{lll}
1 & 1 & 0
\end{array}\right] \rightarrow(1 / 6)\left[\begin{array}{lll}
\overline{1} & 2 & \overline{1}
\end{array}\right]+\left(\begin{array}{lll}
1 / 6)[2 & \overline{1} & 1
\end{array}\right]
\end{aligned}
$$

and

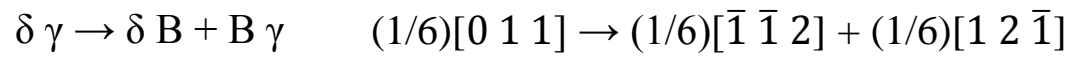

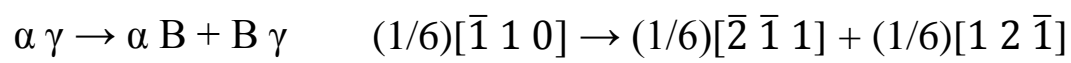



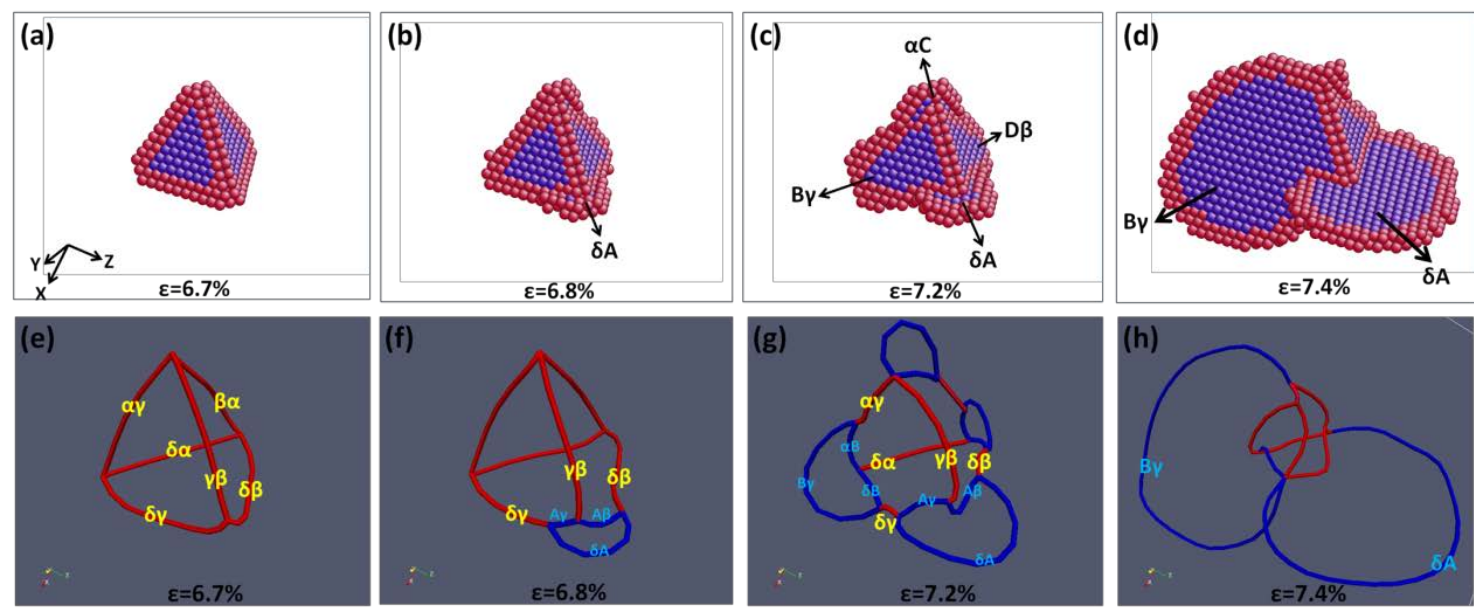

Fig.3 Snapshots of SFT configuration in crystal with $\left[\begin{array}{lll}1 & 0 & 0\end{array}\right]$ orientation at different stages of deformation during uniaxial compression. (a)-(d) shows the results from MD simulation; (e)-(h) shows the extracted dislocation segment by using the dislocation extraction algorithm. The red line represents the stair-rod dislocation and the blue line indicates the Shockley partial dislocation. (Colour online)

Fig.4 shows the evolution of the SFT configuration in crystal with $\left[\begin{array}{lll}1 & 0 & 0\end{array}\right]$ orientation during tensile loading. When the applied tensile strain equaled $\varepsilon=5.5 \%$, the SFT can still retain its original structure as shown in Fig.4(a). It is interesting to find that the SFT structure in equilibrium had changed to another configuration with two unfaulted planes in Fig.4(c) at $\varepsilon=6.2 \%$. The stress-strain curve in this case was checked and revealed that this structural transformation had no effect on the elastic trend and did not contribute to the incipience of plasticity. Moreover, the new structure could now retain its configuration until the tensile loading increased to about $\varepsilon=8.2 \%$. In this study we tentatively called this metastable structure as "semi-faulted SFT". The process of transforming the structure from an SFT to a semi-faulted SFT is shown in Fig.4(e)-(k) based on the dislocation reaction analysis. For a clearer illustration, we indexed the four vertices of SFT as 1, 2, 3 and 4. Initially, four of the SFT stair-rod dislocations $(\delta \beta, \alpha \beta, \delta \gamma$ and $\alpha \gamma)$ tended to dissociate to Shockley partial dislocations from the vertices, as shown in Fig.4(b) and (f) at $\varepsilon=5.8 \%$ : for example, the dissociation at vertex 2 and vertex 3 can be described as:

$$
\begin{aligned}
& \delta \beta \rightarrow \delta C+C \beta \quad(1 / 6)[110] \rightarrow(1 / 6)\left[\begin{array}{lll}
2 & \overline{1} & \overline{1}
\end{array}\right]+(1 / 6)\left[\begin{array}{lll}
\overline{1} & 2 & 1
\end{array}\right]
\end{aligned}
$$

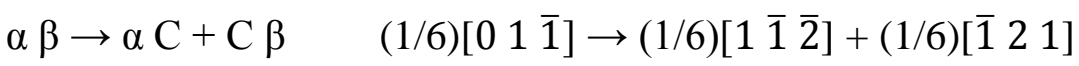

and 


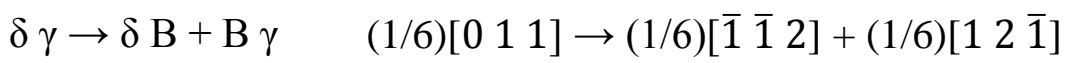

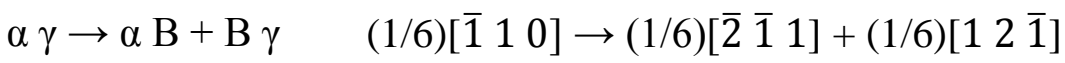

As the tensile deformation increased the partial dislocation $C \beta$ which was dissociated from $\delta \beta$ and $\alpha \beta$ glided on the (1 1 1) plane while the partial dislocation B $\gamma$ which was

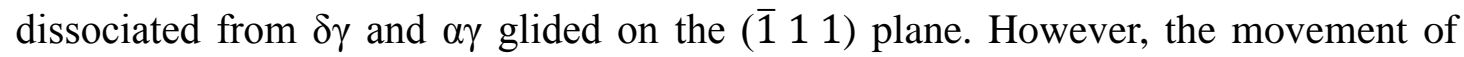
partial dislocations at vertex 1 and vertex 4 were either restrained or even recovered to the initial stair-rod dislocations, as shown in Fig.4(g) and (h) at $\varepsilon=6.065 \%$ and $\varepsilon=6.07 \%$, Fig.4(i) indicates that when the glissile Shockley dislocation C $\beta$ moved forward and reached the edge of SFT, it reacted with the non-dissociated stair-rod dislocation $\gamma \mathrm{B}$ to form a sessile Frank dislocation $\gamma \mathrm{C}$ by the reaction:

$$
\gamma \beta+\beta C \rightarrow \gamma C \quad(1 / 6)[10 \overline{1}]+(1 / 6)[1 \quad \overline{2} \overline{1}] \rightarrow(1 / 3)[1 \quad \overline{1} \overline{1}]
$$

Soon after, the Shockley dislocation $\mathrm{B} \gamma$ from vertex 3 reacted with $\gamma \mathrm{C}$ to form a perfect dislocation $\mathrm{BC}$, as shown in Fig. $4(\mathrm{j})$ at $\varepsilon=6.1 \%$. This reaction can be described as:

$$
\mathrm{B} \gamma+\gamma \mathrm{C} \rightarrow \mathrm{B} \mathrm{C} \quad(1 / 6)[12 \mathrm{1}]+(1 / 3)\left[\begin{array}{lll}
1 & \overline{1} & \overline{1}
\end{array}\right] \rightarrow(1 / 2)\left[\begin{array}{lll}
1 & 0 & \overline{1}
\end{array}\right]
$$

The process of Structural transformation has completed at about $\varepsilon=6.2 \%$. The semi-faulted SFT now consisted of four Shockley partial dislocations $(\delta \mathrm{C}, \alpha \mathrm{C}, \delta \mathrm{B}$ and $\alpha \mathrm{B})$ connected by a stair-rod dislocation $(\delta \alpha)$ and a perfect dislocation (BC). MD simulation indicated that the onset of yielding began because four Shockley partial dislocations propagated from the semi-faulted SFT, as shown in Fig.4(d). 

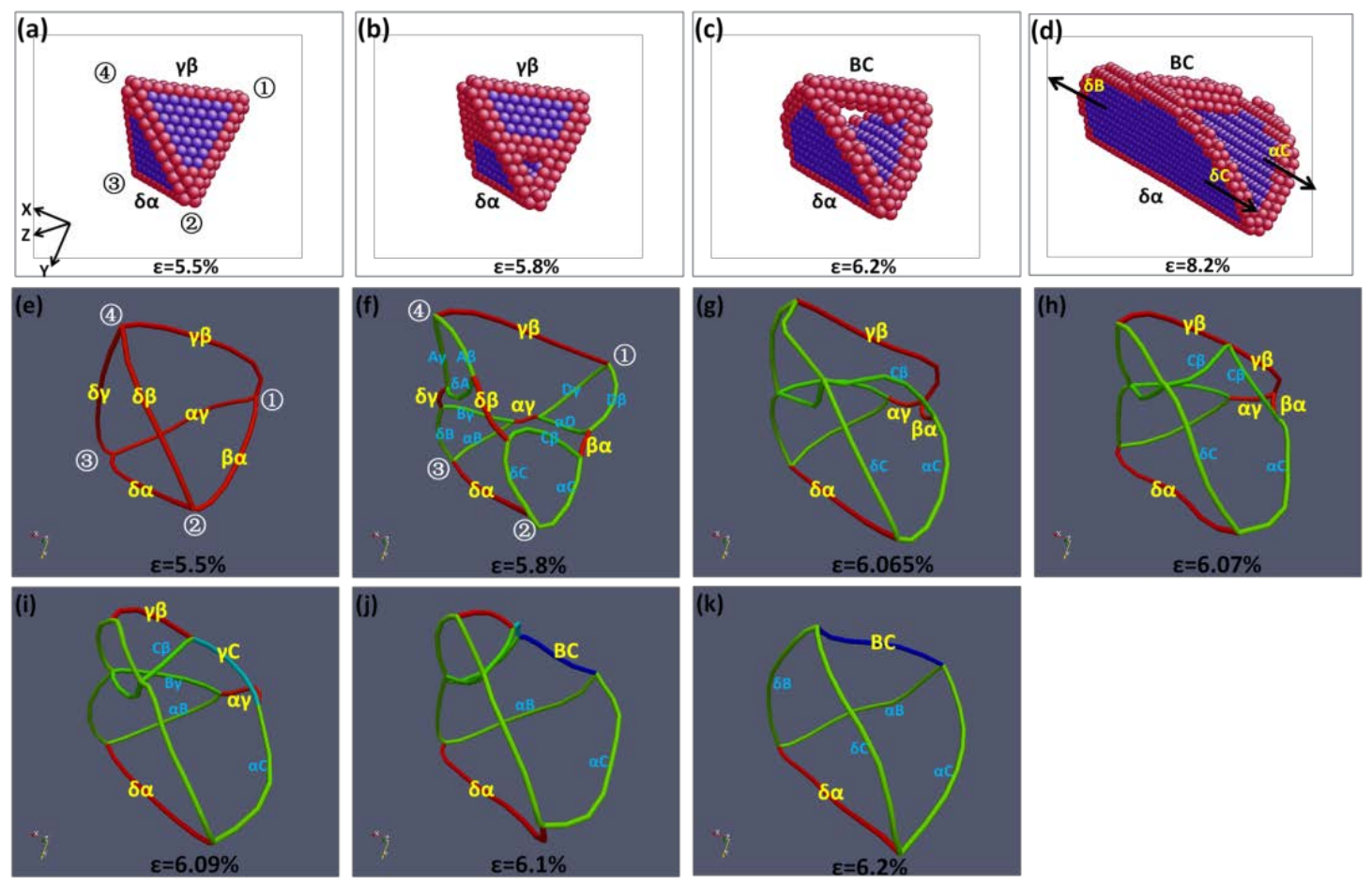

Fig.4 Snapshots of SFT configuration in crystal with [ $\left[\begin{array}{lll}1 & 0 & 0\end{array}\right]$ orientation at different stages of deformation during uniaxial tension. (a)-(d) shows the results from MD simulation; (e)-(k) shows the extracted dislocation segment by using the dislocation extraction algorithm. The red line represents the stair-rod dislocation, the green line represents the Shockley partial dislocation, the light blue line indicates the Frank partial dislocation, and the dark blue line indicates the perfect dislocation. (Colour online)

\subsection{Deformation mechanism of SFT for [ $\left[\begin{array}{lll}1 & 1 & 0\end{array}\right]$ orientation}

Fig.5 shows the SFT configuration in crystal with [1 110$]$ orientation at different stages of deformation during uniaxial compression. As was the case with [1 000$]$ orientation in tension, the equilibrium SFT structure evolved into a metastable structure, i.e. the semi-faulted SFT shown in Fig.5 (b) and (f) at $\varepsilon=4 \%$. However, the Shockley partial dislocations ( $\alpha \mathrm{B}, \gamma \mathrm{B}, \alpha \mathrm{D}$ and $\gamma \mathrm{D})$ involved in the semi-faulted SFT did not propagate with the increased compressive stress, unlike the case of $\left[\begin{array}{lll}1 & 0 & 0\end{array}\right]$ orientation in tension. Fig.5 (c) and (g) indicates that the onset of yielding resulted from the nucleation of a Shockley partial dislocation $\delta \mathrm{B}$ from the perfect dislocation DB in the semi-faulted SFT by the reaction:

$$
\mathrm{D} \mathrm{B} \rightarrow \mathrm{D} \delta+\delta \mathrm{B} \quad(1 / 2)\left[\begin{array}{lll}
1 & 1 & 0
\end{array}\right] \rightarrow(1 / 3)\left[\begin{array}{lll}
1 & 1 & 1
\end{array}\right]+\left(\begin{array}{lll}
1 / 6)[1 & 1 & \overline{2}
\end{array}\right]
$$

Subsequently, the partial dislocation $\delta$ B propagated on the $\left(\begin{array}{lll}1 & 1 & 1\end{array}\right)$ plane and resulted in the crystal plastic deformation shown in Fig.5(d) and (h). This result was consistent 


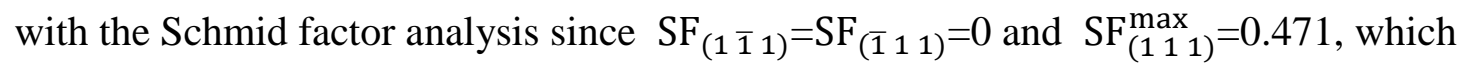
indicates that the gliding of Shockley partial dislocations $(\alpha \mathrm{B}, \gamma \mathrm{B}, \alpha \mathrm{D}$ and $\gamma \mathrm{D})$ on ( $\left.\begin{array}{lll}1 & \overline{1} & 1\end{array}\right)$ and ( $\left.\begin{array}{lll}1 & 1 & 1\end{array}\right)$ planes cannot be activated, while $\delta \mathrm{B}$ slips on $\left(\begin{array}{lll}1 & 1 & 1\end{array}\right)$ plane is the favored slip system.
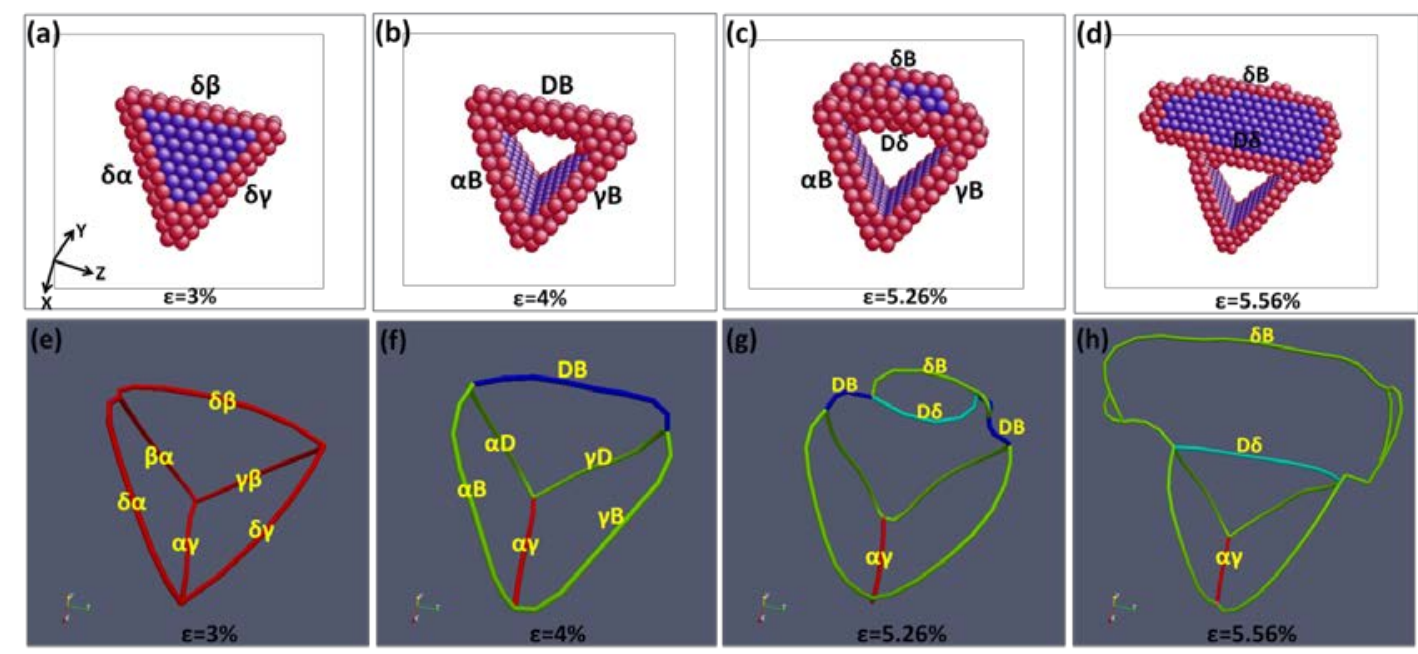

Fig.5 Snapshots of SFT configuration in crystal with [ $\left[\begin{array}{lll}1 & 1 & 0\end{array}\right]$ orientation at different stages of deformation during uniaxial compression. (a)-(d) shows the results from MD simulation; (e)-(h) shows the extracted dislocation segment by using the dislocation extraction algorithm. The red line represents the stair-rod dislocation, the green line represents the Shockley partial dislocation, the light blue line indicates the Frank partial dislocation, and the dark blue line indicates the perfect dislocation. (Colour online)

The snapshots presented in Fig.6 shows the atomistic view of SFT at the onset of yielding (a-c) and the corresponding dislocation mechanism (d-h). When the maximum tensile stress reached about $\varepsilon=4.98 \%$, two stair-rod dislocations ( $\beta \alpha$ and $\gamma \beta$ ) on the $(11 \overline{1})$ plane began to be simultaneously dissociated into Shockley partial dislocations by the reaction:

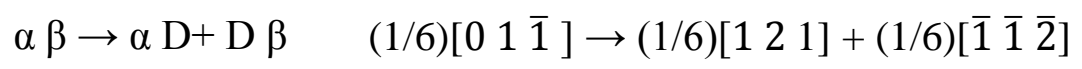

$$
\begin{aligned}
& \gamma \beta \rightarrow \gamma \mathrm{D}+\mathrm{D} \beta \quad(1 / 6)\left[\begin{array}{lll}
1 & 0 & \overline{1}
\end{array}\right] \rightarrow(1 / 6)\left[\begin{array}{lll}
2 & 1 & 1
\end{array}\right]+(1 / 6)\left[\begin{array}{lll}
\overline{1} & \overline{1} & \overline{2}
\end{array}\right]
\end{aligned}
$$

The dissociated Shockley partial D $\beta$ from $\beta \alpha$ and $\gamma \beta$ merged together and propagated as further tensile strain was applied. This process was similar to the case of $\left[\begin{array}{lll}1 & 0 & 0\end{array}\right]$ orientation in compression. However, Fig.6 (e) indicates that the residual Shockley partial dislocations ( $\alpha \mathrm{D}$ and $\gamma \mathrm{D}$ ) neither propagated nor remained stable, instead, they 
split into a stair-rod dislocation and another Shockley partial dislocation $\beta \mathrm{D}$ by the reaction:

$$
\begin{aligned}
& \alpha \mathrm{D} \rightarrow \alpha \beta+\beta \mathrm{D} \quad(1 / 6)\left[\begin{array}{lll}
1 & 2 & 1
\end{array}\right] \rightarrow(1 / 6)\left[\begin{array}{lll}
0 & 1 & \overline{1}
\end{array}\right]+(1 / 6)\left[\begin{array}{lll}
1 & 1 & 2
\end{array}\right] \\
& \gamma \mathrm{D} \rightarrow \gamma \beta+\beta \mathrm{D} \quad(1 / 6)\left[\begin{array}{lll}
2 & 1 & 1
\end{array}\right] \rightarrow(1 / 6)\left[\begin{array}{lll}
1 & 0 & \overline{1}
\end{array}\right]+(1 / 6)\left[\begin{array}{lll}
1 & 1 & 2
\end{array}\right]
\end{aligned}
$$

This step can be regarded as a reverse process of the last step because the original stair-rod dislocation ( $\beta \alpha$ and $\gamma \beta$ ) reverted and the partial dislocation $\beta \mathrm{D}$ had the same Burger vector as $\mathrm{D} \beta$ but it differed in the slip direction. Subsequently, $\beta \mathrm{D}$ propagated on $\left(\begin{array}{lll}1 & 1 & \overline{1}\end{array}\right)$ plane inside the SFT and then reacted with the stair-rod dislocation $\delta \beta$ to form a Frank partial $\delta \mathrm{D}$ and then slipped across $\delta \beta$. This process can be described by the reaction:

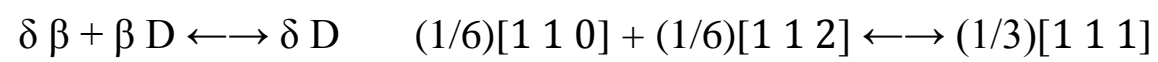

Note that the SFT on ( $\left.\begin{array}{lll}1 & 1 & \overline{1}\end{array}\right)$ plane became unfaulted as $\beta D$ slipped, although this is not shown in the pictures, and as $\beta \mathrm{D}$ traversed, it introduced an extrinsic ledge near the edge of the SFT which caused $\beta$ D to slip on the $\left(\begin{array}{lll}1 & 1 & \overline{1}\end{array}\right)$ plane one layer of atoms higher than $\mathrm{D} \beta$. Since the Shockley partial dislocations $\mathrm{D} \beta$ and $\beta \mathrm{D}$ propagated on the two consecutive (1 $11 \overline{1}$ ) planes, an extrinsic stacking fault was generated inside the partial loop, as shown in Fig.6(c).

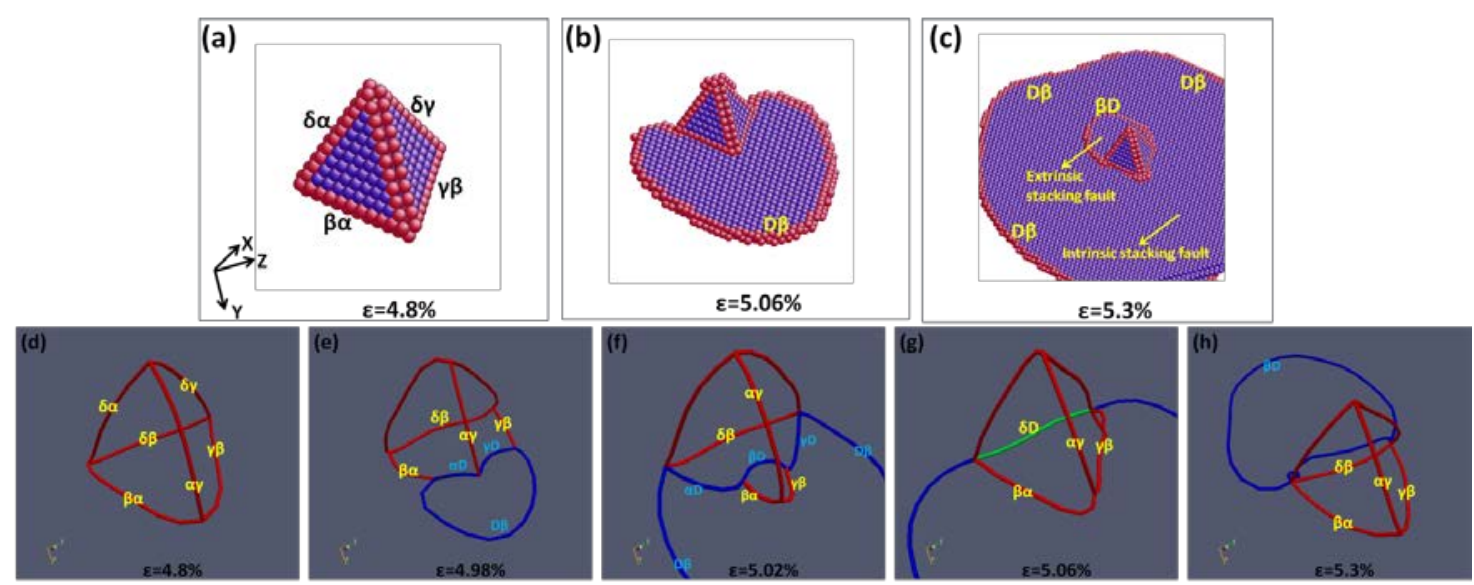

Fig.6 Snapshots of SFT configuration in crystal with [1 110$]$ orientation at different stages of deformation during uniaxial tension. (a)-(c) shows the results from MD simulation; (d)-(h) shows the extracted dislocation segment by using the dislocation extraction algorithm. The red line represents the stair-rod dislocation, the blue line represents the Shockley partial dislocation, and the green line indicates the Frank partial dislocation. (Colour online) 


\subsection{Deformation mechanism of SFT for [ $\left[\begin{array}{lll}1 & 1 & 1\end{array}\right]$ orientation}

The sequence of snapshots presented in Fig.7 shows the SFT configuration at various stages in crystal with [1 111 1] orientation under uniaxial compression where the loading direction was perpendicular to the $\left(\begin{array}{lll}1 & 1 & 1\end{array}\right)$ plane of the tetrahedra. It was interesting to find that the perfect SFT structure gradually collapsed as the compressive deformation increased, and the unzipping of SFT from a single vertex corresponded to the inverse of the Silcox-Hirsch mechanism; this resulted in a perfect SFT being transformed into a triangular Frank loop, as shown in Fig.7(a) and (e).This was consistent with the experiment result[62] where the compressive stress acting on a $\left\{\begin{array}{ll}1 & 1\end{array}\right\}$ plane can promote the formation of faulted loops from vacancy clusters on this plane. By checking the stress-strain curve, the process of structural transformation did not alter the elastic stage. A visual inspection of the MD simulation results indicated that the onset of yielding corresponded to the propagation of a perfect dislocation loop, which poses the key question; how did a sessile Frank partial dislocation loop convert into a perfect glissile dislocation loop? This process is shown in Fig.7(e)-(k).
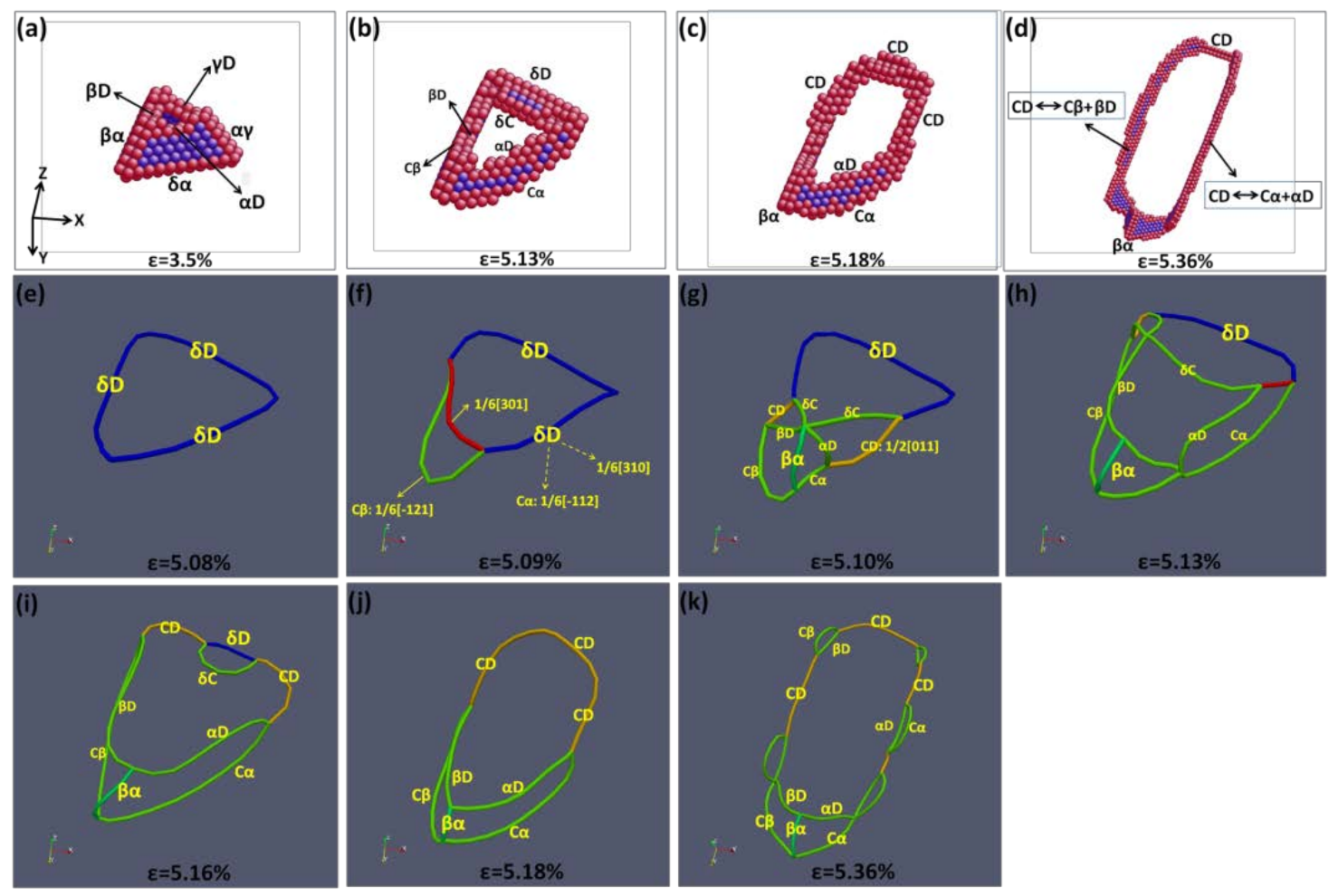

Fig.7 Snapshots of SFT configuration in crystal with [ $\left[\begin{array}{lll}1 & 1 & 1\end{array}\right]$ orientation at different stages of 
deformation during uniaxial compression. (a)-(d) shows the results from MD simulation; (e)-(h) shows the extracted dislocation segment by using the dislocation extraction algorithm. The blue line represents the Frank partial dislocation, the green line represents the Shockley partial dislocation, the yellow line indicates the perfect dislocation, and the red line is the undefined dislocation. (Colour online)

Firstly, as shown in Fig.7(f), one edge of the Frank loop tended to dissociate to a Shockley partial dislocation $C \beta$ and a dislocation with Burgers vector $\mathbf{b}=1 / 6\left[\begin{array}{ll}3 & 0\end{array}\right]$. This was the same for another edge where the Frank dislocation tended to dissociate to $\mathrm{C} \alpha$ and a dislocation with $b=1 / 6\left[\begin{array}{ll}3 & 1\end{array}\right]$, although it is not shown in Fig.7(f). This process can be expressed by:

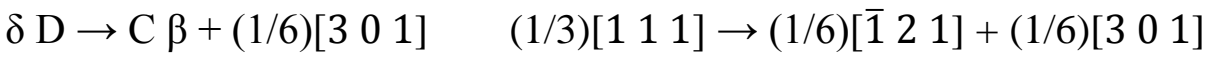

$$
\begin{aligned}
& \delta \mathrm{D} \rightarrow \mathrm{C} \alpha+(1 / 6)\left[\begin{array}{lll}
3 & 1 & 0
\end{array}\right] \quad(1 / 3)\left[\begin{array}{lll}
1 & 1 & 1
\end{array}\right] \rightarrow(1 / 6)\left[\begin{array}{lll}
1 & 1 & 2
\end{array}\right]+\left(\begin{array}{lll}
1 / 6)[3 & 1 & 0
\end{array}\right]
\end{aligned}
$$

Fig.7 (g) shows that when the dissociated segments $C \beta$ and $C \alpha$ met, they formed a stair-rod dislocation $\beta \alpha$ by the reaction:

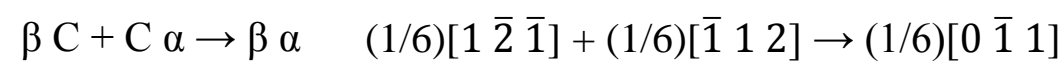

On the other hand the dislocations with $\mathbf{b}=1 / 6\left[\begin{array}{lll}3 & 0 & 1\end{array}\right]$ and $\mathbf{b}=1 / 6\left[\begin{array}{lll}3 & 1 & 0\end{array}\right]$ were quite unstable and soon split into two Shockley partial dislocations respectively, as described by:

$$
\begin{aligned}
& (1 / 6)\left[\begin{array}{lll}
3 & 0 & 1
\end{array}\right] \rightarrow \delta \mathrm{C}+\beta \mathrm{D} \quad(1 / 6)\left[\begin{array}{lll}
3 & 0 & 1
\end{array}\right] \rightarrow(1 / 6)\left[\begin{array}{lll}
2 & \overline{1} & 1
\end{array}\right]+(1 / 6)\left[\begin{array}{lll}
1 & 1 & 2
\end{array}\right]
\end{aligned}
$$

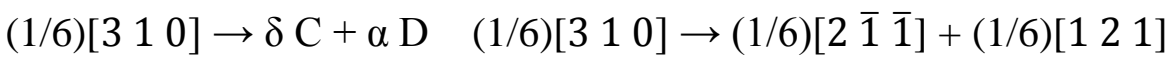

Overall, according to the above reactions we can regard the dissociation process as a Frank partial dislocation that split into three Shockley partial dislocations by the reactions:

$$
\begin{aligned}
& \delta \mathrm{D} \rightarrow \delta \mathrm{C}+\mathrm{C} \beta+\beta \mathrm{D} \\
& (1 / 3)\left[\begin{array}{lll}
1 & 1 & 1
\end{array}\right] \rightarrow(1 / 6)\left[\begin{array}{lll}
2 & \overline{1} & \overline{1}
\end{array}\right]+(1 / 6)\left[\begin{array}{lll}
\overline{1} & 2 & 1
\end{array}\right]+(1 / 6)\left[\begin{array}{lll}
1 & 1 & 2
\end{array}\right]
\end{aligned}
$$

and

$$
\begin{aligned}
& \delta \mathrm{D} \rightarrow \delta \mathrm{C}+\mathrm{C} \alpha+\alpha \mathrm{D} \\
& (1 / 3)\left[\begin{array}{lll}
1 & 1 & 1
\end{array}\right] \rightarrow(1 / 6)\left[\begin{array}{lll}
2 & \overline{1} & 1
\end{array}\right]+(1 / 6)\left[\begin{array}{lll}
\overline{1} & 1 & 2
\end{array}\right]+(1 / 6)\left[\begin{array}{lll}
1 & 2 & 1
\end{array}\right]
\end{aligned}
$$


These simulation results indicated that a partial dislocation $\delta \mathrm{C}$ formed inside the loop and then spread across the loop removing the stacking fault, as shown in Fig.7(b) and (h) at $\varepsilon=5.13 \%$. When $\delta \mathrm{C}$ slipped to the edge of the Frank loop it reacted with the stair-rod dislocation $\delta \mathrm{D}$ to form a perfect dislocation $\mathrm{CD}$, while at the outside the two remaining dissociated Shockley partials ( $C \beta$ and $\beta D, C \alpha$ and $\alpha \mathrm{D})$ reacted with each other to produce a perfect dislocation CD, as shown in Fig.7(i) and (j). Thus, the perfect dislocation loop CD can be generated by the dislocation reactions as:

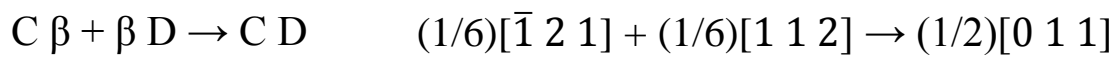

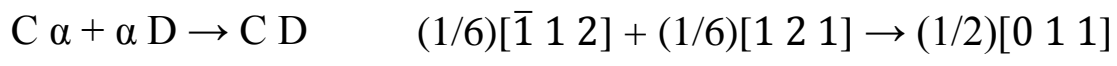

$$
\begin{aligned}
& \mathrm{C} \delta+\delta \mathrm{D} \rightarrow \mathrm{C} \mathrm{D} \quad(1 / 6)\left[\begin{array}{lll}
\overline{2} & 1 & 1
\end{array}\right]+(1 / 3)\left[\begin{array}{lll}
1 & 1 & 1
\end{array}\right] \rightarrow\left(\begin{array}{lll}
1 / 2 & 2
\end{array}\right]\left[\begin{array}{lll}
0 & 1 & 1
\end{array}\right]
\end{aligned}
$$

The dislocation loop expanded rapidly as the compressive stress increased due to the CD slipping until it eventually induced the incipience of a plastic stage. Note that the original Frank loop was on the (1 111 ) plane, while the converted perfect loop was on the $\left(\begin{array}{lll}1 & 1 & \overline{1}\end{array}\right)$ plane. According to the Schmid factor analysis, the perfect dislocation CD

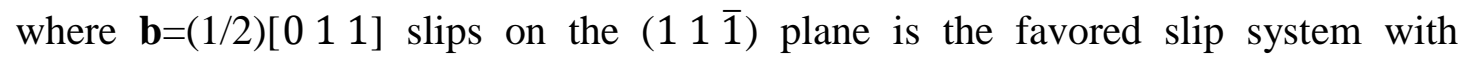
$\mathrm{SF}_{(1 \overline{1} \overline{1})}^{\max }=0.408$. While the CD was slipping, it tended to dissociate back to the initial Shockley partials ( $\mathrm{C} \beta$ and $\beta \mathrm{D}, \mathrm{C} \alpha$ and $\alpha \mathrm{D})$ due to the comparatively low staking fault energy of copper $\left(44.4 \mathrm{~mJ} / \mathrm{m}^{-2}\right)$, as shown in Fig.7(d) and (k). However, the simulation indicated that this was only an oscillating process because the Shockley partial dislocations cannot slip very far away, and the slipping of a perfect dislocation loop still played a dominant role during plastic deformation.

The series of snapshots presented in Fig.8 shows the SFT configuration near the yield point in crystal with [lll $\left.\begin{array}{lll}1 & 1 & 1\end{array}\right]$ orientation during uniaxial tension. When the maximum tensile stress was reached, Fig.8 (b) and (f) shows that a Shockley partial dislocation was dissociated from one edge $(\beta \alpha)$ of the SFT and its reaction was:

$$
\beta \alpha \rightarrow \beta \mathrm{D}+\mathrm{D} \alpha \quad(1 / 6)\left[\begin{array}{lll}
0 & \overline{1} & 1
\end{array}\right] \rightarrow(1 / 6)\left[\begin{array}{lll}
1 & 1 & 2
\end{array}\right]+(1 / 6)\left[\begin{array}{lll}
\overline{1} & \overline{2} & \overline{1}
\end{array}\right]
$$

This was different from the cases in [ $\left[\begin{array}{lll}1 & 0 & 0\end{array}\right]$ orientation in compression and [ $\left.\begin{array}{lll}1 & 1 & 0\end{array}\right]$ orientation in tension, where the partial dislocation was nucleated from the vertex of 
the tetrahedra with two edges of SFT involved. The dissociation of $\beta \alpha$ was completed at $\varepsilon=8 \%$, as shown in Fig. $8(\mathrm{c})$ and (g). Subsequently, the partial dislocation $\beta \mathrm{D}$ and

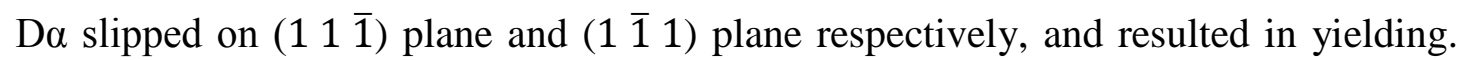
The Schmid factor analysis showed they are the favored slip systems, with $\mathrm{SF}_{(11 \overline{1})}=\mathrm{SF}_{(1 \overline{1} 1)}=0.314$

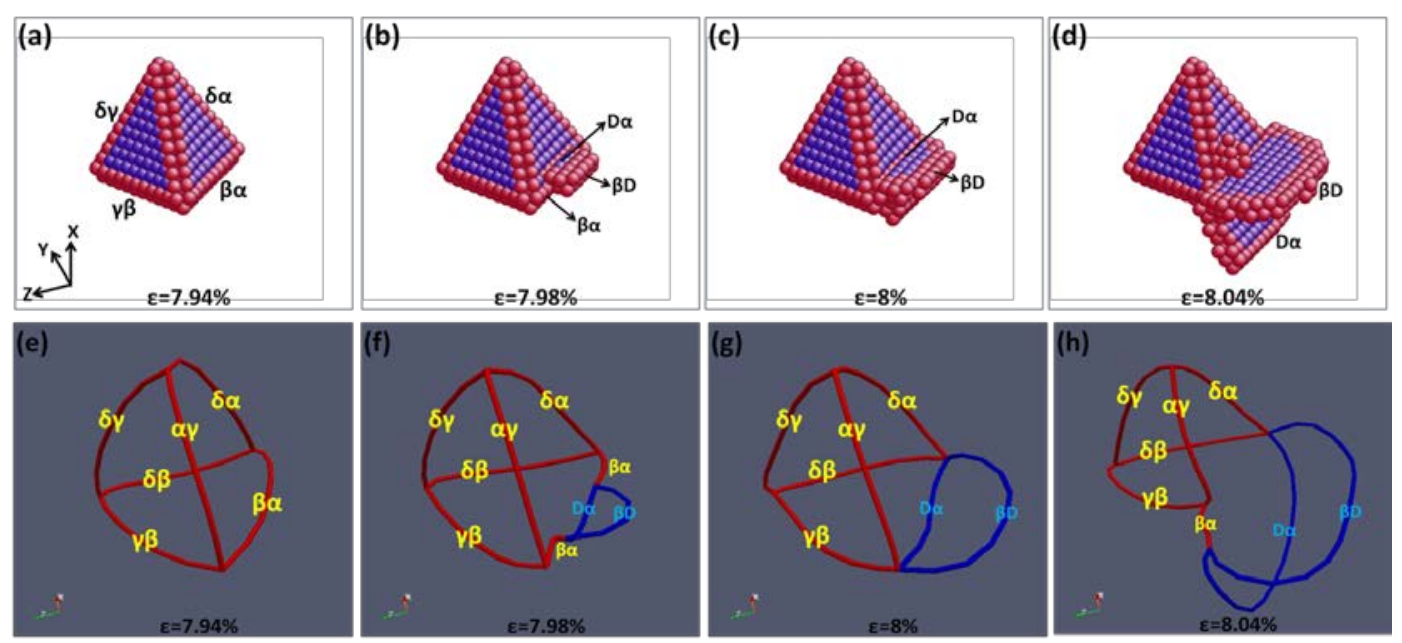

Fig.8 Snapshots of SFT configuration in crystal with [1 1111$]$ orientation at different stages of deformation during uniaxial tension. (a)-(c) shows the results from MD simulation; (d)-(h) shows the extracted dislocation segment by using the dislocation extraction algorithm. The red line represents the stair-rod dislocation, the blue line represents the Shockley partial dislocation. (Colour online)

\section{Discussion}

\subsection{Different reduction in yield stress}

The simulations indicated that the stress in compression and tension needed for plastic deformation was reduced for crystals with SFT in all orientations (see Table.1). This is reasonable because higher stress is required for a homogeneous dislocation from a perfect crystal structure than the heterogeneous dislocation nucleation from an existing structural defect (e.g. SFT in this study). It is worth noting that the reduced yield stress caused by the SFT depended almost entirely on the crystal orientation and loading direction. For example, the reduced yield stress in compression is less than in tension for the [ $\left.\begin{array}{lll}1 & 0 & 0\end{array}\right]$ orientation, at $24.9 \%$ and $30.8 \%$ respectively. However, this reduction seems to be much higher in compression (37.1\% and 57.3\%) than in tension (15.8\% and 19.3\%) for [ $\left.\begin{array}{llll}1 & 1 & 0\end{array}\right]$ and [ $\left.1 \begin{array}{lll}1 & 1 & 1\end{array}\right]$ orientation. 

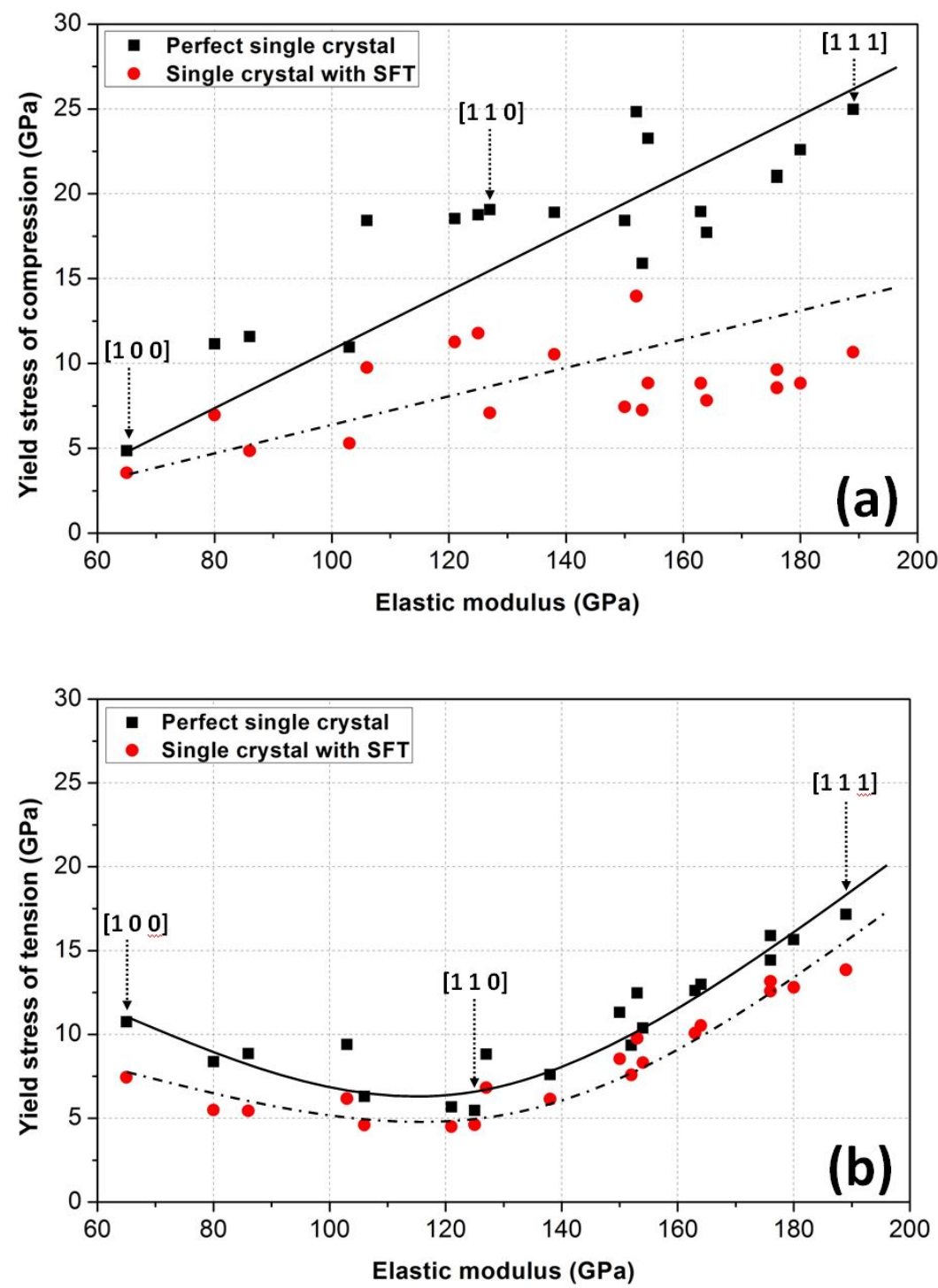

Fig.9 Yield stresses of perfect single crystals and crystals with SFT as a function of elastic modulus for various of crystal orientations under (a) uniaxial compression and (b) uniaxial tension. The solid line and the dash-dot line are draw to guide the eye.

Simulations revealed that copper has a high degree of elastic anisotropy depending on the loading axis orientation. For example, the calculated elastic modulus of [1 1111$]$ orientation $\left(\mathrm{E}_{[111]}=189 \mathrm{GPa}\right)$ is almost three times greater than the value of $\left[\begin{array}{lll}1 & 0 & 0\end{array}\right]$ orientation $\left(\mathrm{E}_{[100]}=65 \mathrm{GPa}\right)$. Therefore, it is important to examine the influence of the elastic modulus on the simulation results. Fig.9 plots the yield stress of perfect single crystals and crystals with SFT as a function of the elastic modulus. In compression, the yield stress generally increases with the increased elastic modulus for perfect single crystals, whereas in the presence of SFT, the overall yield stress also increases as a function of the increasing elastic modulus, but the influence of elastic modulus on 
the yield stress is less evident than for the perfect crystal. For example, the maximum yield stress (13.97 GPa) in compression was observed in the [8 7 1] orientation, which has only a moderate elastic modulus $\left(\mathrm{E}_{[871]}=152 \mathrm{GPa}\right)$. In tension, the elastic modulus has less influence on the yield stress when the value is less than $120 \mathrm{GPa}$, while there is an obvious increase in the yield stress at a higher elastic modulus. Fig.10 shows the reduction in the yield stresses due to the presence of SFT as a function of elastic modulus for different crystal orientations. It can be seen that the elastic modulus can plays a major role in the different reduction of yield stresses because the reduction is more significant for stiffer orientations in compression while an opposite trend is seen in tension. However, the scattered points (e.g. [8 2 1] 1 and [4 2 1] orientation) indicates that the elastic modulus is insufficient to explain the different reduction in yield stress between compression and tension.

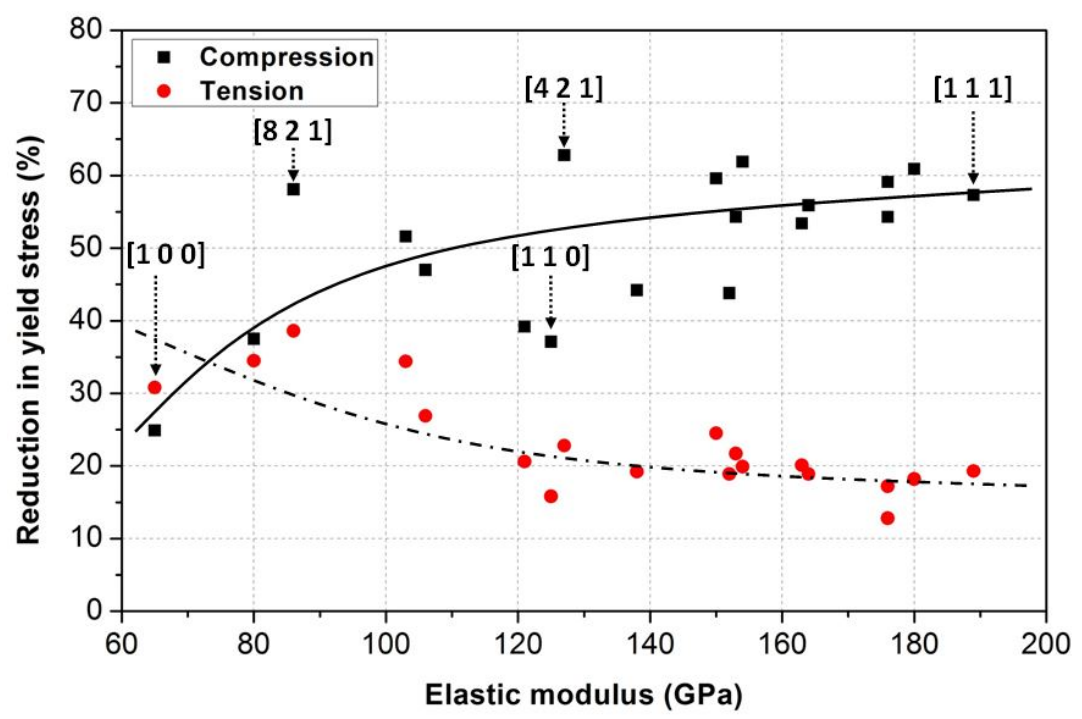

Fig.10 The reduction in yield stresses under uniaxial compression and tension as a function of elastic modulus for various of crystal orientations. The solid line and the dash-dot line are draw to guide the eye.

A visual inspection of the MD simulation revealed that the difference of yield stress reduction can be attributed to the different mechanisms between compression and tension at the incipience of plastic deformation. For example, for [ 100 l 0 orientation in

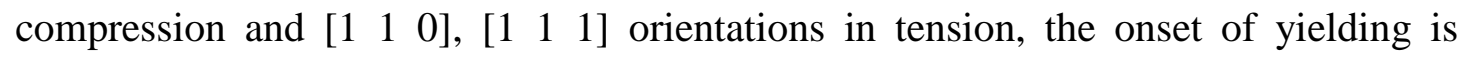
resulted from the dislocations nucleation from the edge of SFT, as shown in Fig.2 (a), 
(d) and (f). The detailed nucleation processes are shown in Fig.3, Fig.6 and Fig.8

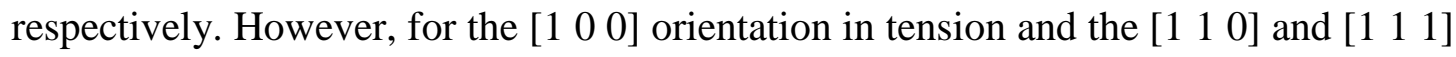
orientations in compression, an obvious structural transformation occurs before dislocation propagation, although this process is still in the elastic stage and has almost no effect on the stress-strain curve. The transformation of a perfect SFT resulted in the formation of a metastable semi-faulted SFT in the cases of [ $\left[\begin{array}{lll}1 & 0 & 0\end{array}\right]$ and [1 11 0] orientation (see Fig.4 and Fig.5), while for the [1 111 1] orientation, the perfect 3D SFT evolved to a 2D Frank loop (see Fig.7). The structural transformation is a stress-assisted process, and the transformed structures have higher energy than the initial perfect SFT. From the perspective of energy, the structures with higher energy can facilitate the dislocation nucleation, which makes the incipient plastic deformation easier than the original perfect SFT, and therefore the relative change of stress required to trigger plastic deformation is greater in crystals with the transformed SFT. Thus, the reduced yield stress is more evident in the cases where structural transformation occurred during loading. In particular, the collapse of an SFT during compressive loading is not always carried out simultaneously on the three stacking fault planes from a single vertex. The unzipping of an SFT can be completed in only one or two stacking fault planes depending on the different crystal orientations, which results in the nucleation of a single or double perfect dislocation loops. The partial unzipping of SFT will be discussed elsewhere.

\subsection{Compression/tension asymmetry}

The simulation revealed a distinct $\mathrm{C} / \mathrm{T}$ asymmetry for perfect crystals and crystals with SFT, i.e. there was a difference in the yield stress between compression and tension. The C/T asymmetry ratio is plotted as a function of elastic modulus in Fig.11. For the perfect single crystals, elastic hardening in compression can result in a higher yield stress than in tension that usually exhibits an elastic softening. Therefore, most of the asymmetry values for the perfect single crystals are larger than unity, which represents a higher yield strength in compression than in tension. The [1 110$]$ orientation shows the highest C/T ratio (3.42). The [ $\left[\begin{array}{lll}1 & 0 & 0\end{array}\right]$ orientation is a special case 
with a C/T ratio of less than unity (0.45), indicating a higher tensile stress than the compressive stress. Compared to the perfect crystals, the $\mathrm{C} / \mathrm{T}$ asymmetry can be decreased or even reversed in the presence of SFT. For example, the C/T asymmetry ratio decreased from 3.42 to 2.56 for the [ $\left.\begin{array}{lll}1 & 1 & 0\end{array}\right]$ orientation, indicating a decreased C/T asymmetry. Moreover, this ratio decreases from 1.45 to 0.77 for the $\left[\begin{array}{lll}1 & 1 & 1\end{array}\right]$ orientation, which signifies that the $\mathrm{C} / \mathrm{T}$ asymmetry had reversed due to the presence of SFT. Almost half of the crystals with SFT in this study showed a C/T ratio that very close to or less than unity, as shown in Fig.12. Recall that the crystal under compression shows a higher reduction in yield stress than under tension for almost all orientations, and this deviation seemed more obvious with the increased elastic modulus, which eventually caused an overall decreased C/T ratio. Moreover, the MD simulation indicated that the generation of a perfect dislocation loop during loading can be an important reason why the C/T asymmetry reversed. In the cases of [1 00 l orientation in tension and [1 110$]$ orientation in compression, the transformed semi-faulted SFT was a metastable structure that can steadily exist in the crystal during an applied load. A higher stress was required to drive the dislocation nucleation from it and induce the subsequent yielding, as shown in Fig.4 and Fig.5. However, with the [lll $\left.\begin{array}{lll}1 & 1 & 1\end{array}\right]$ orientation during compression, the Frank loop that transformed from an unzipped SFT was quite unstable and soon converted to a perfect dislocation loop. Compared to a sessile Frank loop, the perfect loop is easy to propagate because on a small increase in stress can make it move and induce the onset of plastic deformation. The stress needed for dislocation propagation is much less than that needed for dislocation nucleation. Therefore, the reduction in the yield stress

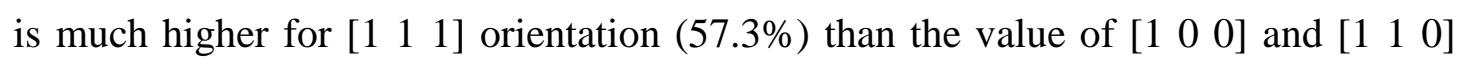
orientation (30.8\% and 37.1\% respectively), where the dislocation was nucleated from the semi-faulted SFT. The sharp decline in compressive stress due to the propagation of a perfect dislocation loop caused the C/T asymmetry to reverse in the $\left[\begin{array}{lll}1 & 1 & 1\end{array}\right]$ orientation crystal. By checking the simulation results, the perfect dislocation loop that generated from a full unzipped or partial unzipped SFT can generally result in a much higher reduction in the compressive stress. 


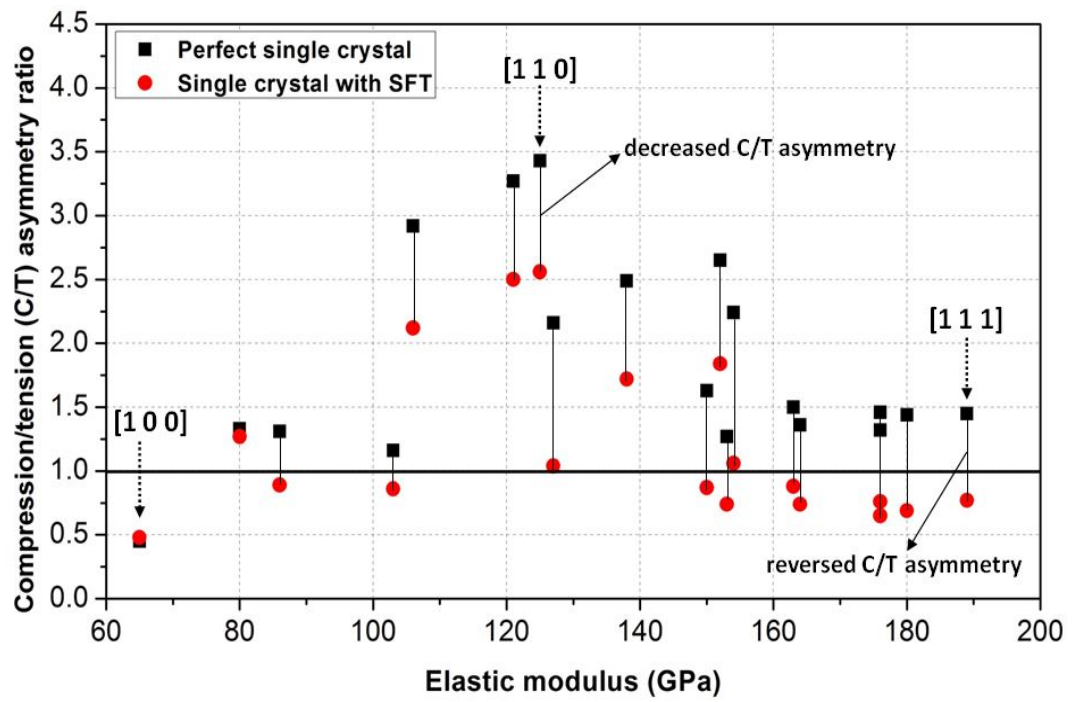

Fig.11 C/T asymmetry ratio of perfect crystal and crystal with SFT as a function of elastic modulus for different crystal orientations.

\subsection{Unfaulting of the Frank loop}

A similar case of structural conversion from a Frank loop to a perfect loop was reported in previous experiments[33, 63, 64]. For example, Westmacott et al.[63] observed a large number of Frank dislocation loops in an aluminum 3.5\% magnesium alloy quenched from $550^{\circ} \mathrm{C}$ into oil at $-20^{\circ} \mathrm{C}$. After being heated slightly, some of the Frank loops were unfaulted and converted to perfect dislocation loops. Note that the conversion of a Frank partial loop to a perfect dislocation loop was probably a spontaneous process in their experiment because this conversion occurred in a stress free condition and the only inducement was the slightly increased temperature. However, the conversion in this study was determined as a stress assisted process. In the case of [ [ $\left.\begin{array}{lll}1 & 1 & 1\end{array}\right]$ crystal orientation, a 5\% compressive strain, according to $10 \mathrm{GPa}$ compressive stress, was needed to achieve this conversion. Recall that, after a Frank loop was generated from a vacancy cluster, it spontaneously dissociated to an SFT through the Silcox-Hirsch mechanism (see Fig.1) instead of converting to a perfect dislocation loop. Therefore, the critical questions are: what are the conditions in which an unfaulting Frank loop tends to occur, and in what conditions is an SFT unlikely to be produced from a vacancy cluster?

As Fig.7 shows, the unfaulting process was initiated from the dissociation of the Frank partial dislocation loop. This process was evidenced by the nucleation of 
Shockley partial dislocations from the stair-rod dislocation (see Fig.7(f)) and the subsequent gliding of one Shockley partial dislocation across the stacking fault. The principle problem is whether or not the prevailing condition can cause the Shockley partial dislocation to nucleate and then spread across the stacking fault in the Frank loop. From the perspective of energy, if the unfaulting process can be completed spontaneously, a necessary condition is that the perfect dislocation loop has a lower energy value than the that of the initial Frank loop, i.e. the removing of the stacking fault from the loop should have reduced the system energy. According to the elasticity theory of dislocation[58, 59], the dislocation can be regarded as being created by elastically deforming an initially unstrained hollow cylinder of unit length and having an outside radius $R$ and inside radius $r_{0}$. The strain energy per unit length of dislocation is:

$$
\begin{gathered}
\mathrm{E}_{\mathrm{el}(\text { screw })}=\frac{\mathrm{Gb}^{2}}{4 \pi} \ln \left(\frac{\mathrm{R}}{\mathrm{r}_{0}}\right) \\
\mathrm{E}_{\mathrm{el}(\text { edge })}=\frac{\mathrm{Gb}^{2}}{4 \pi(1-v)} \ln \left(\frac{\mathrm{R}}{\mathrm{r}_{0}}\right)
\end{gathered}
$$

where $G$ is the shear modulus, $v$ is Poisson's ratio, $b$ is the magnitude of the Burgers vector. $r_{0}$ implies the radius of the dislocation core. To a good approximation, the line length of a dislocation loop was noted by $2 \pi \mathrm{r}$, the elastic energy of a circular edge loop in an isotropic solid with Burgers vector $\mathbf{b}_{\mathbf{e}}$ perpendicular to the loop plane is:

$$
E=\frac{G b_{e}^{2} r}{2(1-v)} \ln \left(\frac{2 r}{r_{0}}\right)
$$

and for a circular shear loop with Burgers vector $\mathbf{b}_{\text {s }}$ lying in the loop plane, the elastic energy is:

$$
\mathrm{E}=\frac{\mathrm{Gb}_{\mathrm{s}}^{2} \mathrm{r}}{2(1-v)}\left(1-\frac{v}{2}\right) \ln \left(\frac{2 \mathrm{r}}{\mathrm{r}_{0}}\right)
$$

The shear loop is a mixture type of dislocation with both edge and screw characters. On opposite sides of a loop, the stress fields of dislocation segments will tend to 
counteract at a distance of about $2 \mathrm{r}$ from the loop, so that the value of outside radius $\mathrm{R}$ is approximately equal to 2r. For the Frank partial dislocation loop with $b=1 / 3[111]$, $\mathrm{b}_{\mathrm{e}}^{2}=\mathrm{a}^{2} / 3$ and $\mathrm{b}_{\mathrm{s}}^{2}=0$, and for the perfect dislocation loop with $\mathrm{b}=1 / 2\left[\begin{array}{lll}1 & 1 & 0\end{array}\right], \mathrm{b}_{\mathrm{e}}^{2}=\mathrm{a}^{2} / 3$ and $b_{\mathrm{s}}^{2}=\mathrm{a}^{2} / 6$. Thus, the difference in energy between the perfect, unfaulted dislocation loop and the initial Frank partial dislocation loop containing the stacking fault is:

$$
\Delta \mathrm{E}=\pi \mathrm{r}^{2} \gamma-\frac{\mathrm{Ga}^{2} \mathrm{r}}{24}\left(\frac{2-v}{1-v}\right) \ln \left(\frac{2 \mathrm{r}}{\mathrm{r}_{0}}\right)
$$

Therefore, the process of unfaulting will be energetically favorable if:

$$
\gamma>\frac{\mathrm{Ga}^{2}}{24 \pi \mathrm{r}}\left(\frac{2-v}{1-v}\right) \ln \left(\frac{2 \mathrm{r}}{\mathrm{r}_{0}}\right)
$$

This equation indicates that the removal of a fault in a Frank partial loop depends on the size of the dislocation loop (r) and the stacking fault energy $(\gamma)$. For a certain size Frank loop, the equation gives a lower limit to the value of $\gamma$ for the removal of a fault. In the experiment with aluminum alloy by Westmacott et al.[63], taking a $=0.405 \mathrm{~nm}$ (lattice constant of aluminum), $\mathrm{r}=10 \mathrm{~nm}$ (which is close to the minimum size of electron microscope for resolving loops), $v=0.33, r_{0}=0.5 \mathrm{~nm}$ and $\mathrm{G}=30 \mathrm{GN} \mathrm{m}^{-2}$, the critical stacking fault energy was calculated to be almost $60 \mathrm{~mJ} \mathrm{~m}^{-2}$, which was much less than the stacking fault energy of aluminum (about $146 \mathrm{~mJ} \mathrm{~m}^{-2}$ [48]). So that the Frank loops tended to convert to perfect loops in their experiment is reasonable, but in this study, by taking a $=0.361 \mathrm{~nm}$ (lattice constant of copper), $\mathrm{r}=2 \mathrm{~nm}$ (considering the size of the introduced vacancy cluster and converted perfect loop), $v=0.33, \mathrm{r}_{\mathrm{o}}=0.5 \mathrm{~nm}$ and $\mathrm{G}=45 \mathrm{GN} \mathrm{m}{ }^{-2}$, the critical stacking fault energy was calculated to be almost 202 $\mathrm{mJ} \mathrm{m}^{-2}$. This was much higher than the stacking fault energy of copper (about $44.4 \mathrm{~mJ}$ $\mathrm{m}^{-2}$ [48]), and therefore the Frank loop tended to be dissociated to an SFT with a lower energy instead of converting to a perfect loop. However, an MD simulation for the [1 11 1] orientation in compression implies that a concentration of local stress can promote this conversion.

\section{Conclusions}

Molecular dynamics simulations were carried out to investigate the effect of 
stacking fault tetrahedron on the incipience of plasticity in copper single crystal with different orientations under uniaxial compression and tension. Three crystal

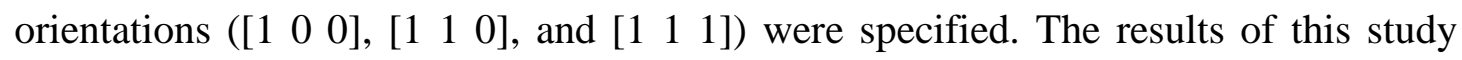
presented a detail view of the underlying deformation mechanisms of SFT induced plasticity in small volume crystals. The main conclusions can be summarized as follows:

(1) The incipience of plasticity in crystals with an SFT resulted from the heterogeneous dislocation nucleation from SFT, while homogeneous dislocation nucleation accounted for the onset of plasticity in the perfect crystals. Therefore, the stress needed for plastic deformation in the crystals with an SFT was less than that required for the perfect crystals in all cases. This result implies that a more strict test standard should be carried out when testing materials that will be used in some extreme conditions, e.g. materials exposed in a radiation environment where a large amount of SFT exists.

(2) The structural transformation of SFT was frequent under applied loading. A metastable structure, i.e. the semi-faulted SFT, was found in the cases of [ $\left[\begin{array}{lll}1 & 0 & 0\end{array}\right]$

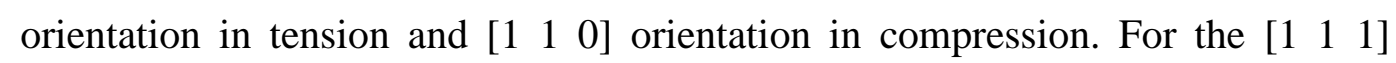
orientation, the SFT tended to collapse to a Frank loop and eventually converted to a perfect dislocation loop under compression. The stress required for dislocation nucleation from a transformed SFT structure was much less than from a perfect SFT structure, which resulted in a different reduction in yield stress for different crystal orientations and loading directions.

(3) The reduction in yield stress due to the presence of SFT was higher in compression than in tension for almost all orientations, and this difference was more evident with the increased elastic modulus. This reduction in yield stress can lead to a decreased or even reversed compression/tension asymmetry, suggesting that both of the tension and compression tests are necessary to prove the mechanical property of materials where SFT are easy to form. The compression test is more reliable because it can usually induce a higher reduction of the yield strength than in tension. 
(4) Simulation results indicated that compressive loading can usually result in the collapse of SFT and facilitate its conversion to a perfect dislocation loop. According to the elastic theory of dislocation, the size of a dislocation loop and the stacking fault energy play a significant role in the formation of SFT and the unfaulting of Frank loop to form a perfect dislocation loop.

\section{Acknowledgements}

This work was supported by an Australian Research Council Discovery Projects (DP130103973). Simulations were performed using the HPC cluster of University of Wollongong and the computing facilities provided by NCI National Facility of Australia. L.Z., X.Z and L.P would like to acknowledge financial support from China Scholarship Council (CSC). 


\section{References}

[1] K.S. Kumar, H. Van Swygenhoven, S. Suresh, Acta Materialia, 51 (2003) 5743-5774.

[2] M.A. Meyers, A. Mishra, D.J. Benson, Progress in Materials Science, 51 (2006) 427-556.

[3] H. Van Swygenhoven, J.R. Weertman, Materials Today, 9 (2006) 24-31.

[4] D. Wolf, V. Yamakov, S.R. Phillpot, A. Mukherjee, H. Gleiter, Acta Materialia, 53 (2005) 1-40.

[5] D. Farkas, Current Opionion in Solid State \& Materials Science, 17 (2013) 284-297.

[6] X. Xiao, D. Song, J. Xue, H. Chu, H. Duan, International Journal of Plasticity, 65 (2014) 152-167.

[7] M.A. Tschopp, D.E. Spearot, D.L. McDowell, Modelling and Simulation in Materials Science and Engineering, 15 (2007) 693-709.

[8] G.E. Norman, A.V. Yanilkin, Phys. Solid State, 53 (2011) 1614-1619.

[9] T.Y. Tan, Philosophical Magazine A, 44 (1981) 101-125.

[10] L.H. Su, C. Lu, L.Z. He, L.C. Zhang, P. Guagliardo, A.K. Tieu, S.N. Samarin, J.F. Williams, H.J. Li, Acta Materialia, 60 (2012) 4218-4228.

[11] V.S. Krasnikov, A.E. Mayer, International Journal of Plasticity, 74 (2015) 75-91.

[12] L. Pei, C. Lu, X. Zhao, L. Zhang, K. Cheng, G. Michal, K. Tieu, Acta Materialia, 89 (2015) 1-13.

[13] S. Brochard, N. Junqua, J. Grilhé, Philosophical Magazine A, 77 (1998) 911-922.

[14] B. Roos, B. Kapelle, G. Richter, C.A. Volkert, Applied Physics Letters, 105 (2014) 201908.

[15] D.E. Spearot, K.I. Jacob, D.L. McDowell, International Journal of Plasticity, 23 (2007) 143-160.

[16] M.A. Tschopp, D.L. McDowell, International Journal of Plasticity, 24 (2008) 191-217.

[17] G.J. Tucker, D.L. McDowell, International Journal of Plasticity, 27 (2011) 841-857.

[18] L. Zhang, C. Lu, K. Tieu, Scientific Reports, 4 (2014).

[19] L. Zhang, C. Lu, K. Tieu, X. Zhao, L. Pei, Nanoscale, 7 (2015) 7224-7233.

[20] J. Silcox, P.B. Hirsch, Philosophical Magazine, 4 (1959) 72-89.

[21] M.H. Loretto, L.M. Clarebrough, R.L. Segall, Philosophical Magazine, 11 (1965) 459-465.

[22] M. Kiritani, J.i. Takamura, M. Doyama, Point defects and defect interactions in metals, University of Tokyo Press, Amsterdam, 1982.

[23] P.J. Phillips, M.J. Mills, Philosophical Magazine, 93 (2013) 82-95.

[24] B.D. Wirth, V. Bulatov, T. Diaz de la Rubia, Journal of Nuclear Materials, 283 (2000) 773-777.

[25] Y.N. Osetsky, D.J. Bacon, B.N. Singh, B. Wirth, Journal of Nuclear Materials, 307 (2002) 852-861.

[26] H. Wang, D.S. Xu, R. Yang, P. Veyssière, Acta Materialia, 59 (2011) 1-9.

[27] H. Wang, D.S. Xu, R. Yang, P. Veyssière, Acta Materialia, 59 (2011) 10-18.

[28] H. Wang, D.S. Xu, R. Yang, P. Veyssière, Acta Materialia, 59 (2011) 19-29.

[29] M.H. Loretto, P.J. Phillips, M.J. Mills, Scripta Materialia, 94 (2015) 1-4.

[30] Y. Matsukawa, S.J. Zinkle, Science, 318 (2007) 959-962.

[31] B.D. Wirth, V.V. Bulatov, T. De La Diaz Rubia, Journal of Engineering Materials and Technology, 124 (2002) 329-334.

[32] Y.N. Osetsky, R.E. Stoller, D. Rodney, D.J. Bacon, Materials Science and Engineering A, 400-401 (2005) 370-373.

[33] J.S. Robach, I.M. Robertson, H.J. Lee, B.D. Wirth, Acta Materialia, 54 (2006) 1679-1690.

[34] L. Saintoyant, H.-J. Lee, B.D. Wirth, Journal of Nuclear Materials, 361 (2007) 206-217.

[35] H.J. Lee, B.D. Wirth, Philosophical Magazine, 89 (2009) 821-841.

[36] M. Niewczas, R.G. Hoagland, Philosophical Magazine, 89 (2009) 623-640. 
[37] D. Kiener, P. Hosemann, S.A. Maloy, A.M. Minor, Nature Materials, 10 (2011) 608-613.

[38] J. Wei Wang, S. Narayanan, J. Yu Huang, Z. Zhang, T. Zhu, S.X. Mao, Nature Communications, 4 (2013) 2340.

[39] M. Niewczas, R.G. Hoagland, Philosophical Magazine, 89 (2009) 727-746.

[40] K.Y. Yu, D. Bufford, C. Sun, Y. Liu, H. Wang, M.A. Kirk, M. Li, X. Zhang, Nature Communications, 4 (2013) 1377.

[41] X. Xiao, D. Song, H. Chu, J. Xue, H. Duan, International Journal of Plasticity, 74 (2015) 110-126.

[42] E. Martinez, J. Marian, A. Arsenlis, M. Victoria, J.M. Perlado, Philosophical Magazine, 88 (2008) 809-840.

[43] E. Martinez, J. Marian, J.M. Perlado, Philosophical Magazine, 88 (2008) 841-863.

[44] I. Salehinia, D.F. Bahr, Scripta Materialia, 66 (2012) 339-342.

[45] I. Salehinia, S.K. Lawrence, D.F. Bahr, Acta Materialia, 61 (2013) 1421-1431.

[46] I. Salehinia, D.F. Bahr, International Journal of Plasticity, 52 (2014) 133-146.

[47] S. Plimpton, Journal of Computational Physics, 117 (1995) 1-19.

[48] Y. Mishin, D. Farkas, M.J. Mehl, D.A. Papaconstantopoulos, Physical Review B, 59 (1999) 3393-3407.

[49] L. Zhang, C. Lu, K. Tieu, X. Zhao, L. Pei, G. Michal, Chinese Physics B, 24 (2015) 88106-088106.

[50] Y. Mishin, M.J. Mehl, D.A. Papaconstantopoulos, A.F. Voter, J.D. Kress, Physical Review B, 63 (2001) 2241061-22410616.

[51] J. Li, Modelling and Simulation in Materials Science and Engineering, 11 (2003) 173.

[52] A. Stukowski, Modelling and Simulation in Materials Science and Engineering, 18 (2010) 015012-015012 (015017).

[53] J. Schiotz, F.D. Di Tolla, K.W. Jacobsen, Nature, 391 (1998) 561-563.

[54] A. Stukowski, K. Albe, Modelling and Simulation in Materials Science and Engineering, 18 (2010) 085001.

[55] A. Stukowski, Modelling and Simulation in Materials Science and Engineering, 20 (2012) 045021.

[56] Y. Dai, M. Victoria, Acta Materialia, 45 (1997) 3495-3501.

[57] Y. Dai, M. Victoria, in: Materials Research Society Symposium Proceedings, 1997, pp. 319-324.

[58] D. Hull, D. Bacon. Introduction to Dislocations (Fifth Edition). 2011.

[59] J.P. Hirth. Theory of Dislocations (Second Edition). 1982.

[60] D.E. Spearot, M.A. Tschopp, D.L. McDowell, Scripta Materialia, 60 (2009) 675-678.

[61] M.A. Tschopp, D.L. McDowell, Journal of the Mechanics and Physics of Solids, 56 (2008) 1806-1830.

[62] A. Sato, Y. Sugisaki, T. Mori, Acta Metallurgica, 31 (1983) 805-811.

[63] K.H.Westmacott, R.S.Barnes, D.Hull, R.E.Smallman, Philosophical magazine, 6 (1961) 929.

[64] S. Yoshida, Journal of the Physical Society of Japan, 18 (1963) 175-183. 